The Free Internet Journal

for Organic Chemistry
Review

Arkivoc 2017, part i, 402-435

\title{
cine- and tele-Substitution reactions: review of work from 2002-2016
}

\author{
Jerzy W. Suwiński
}

Centre of Polymer and Carbon Materials, Polish Academy of Sciences,

M. Skłodowskiej-Curie 34, Zabrze 41-819

E-mail: jsuwinski@cmpw-pan.edu.pl

Received 06-09-2017

Accepted 06-28-2017

Published on line 10-15-2017

\section{Abstract}

This report brings new facts and achievements on the cine- and tele-substitutions reactions published in the last fifteen years. The reactions occurred primarily in such systems as nitroarenes, metal homoarene complexes, and five- or six-membered heteroarenes. Sometimes similar reactions were also observed in other systems, including non-aromatic. cine- and tele-Substitution reactions are presented here independently of their mechanisms.
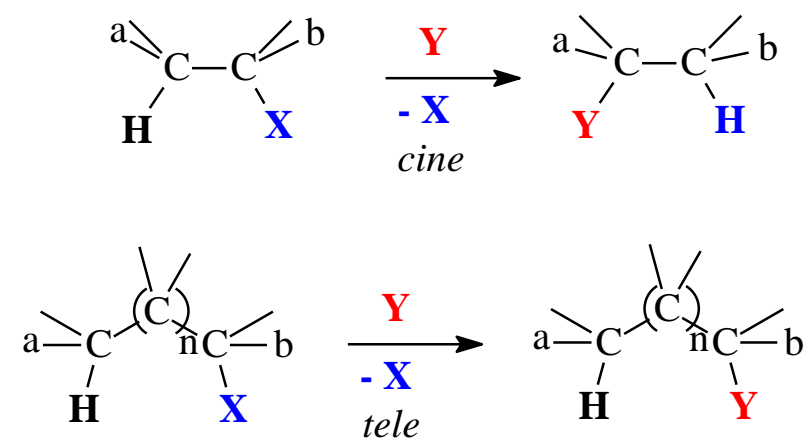

Keywords: Substitution, cine, tele, arene, complex 


\section{Table of Contents}

1. Introduction

2. cine- and tele-Substitution of Homoarene Derivatives

2.1. Nitrohomoarenes

2.2. Complexes of homoarenes with metals

2.3. Other homoarene derivatives

3. cine- and tele-Substitution of Heteroarene Derivatives

3.1 Six-membered heterocycles with a leaving group at a carbon atom

3.2. Five-membered heterocycles with a leaving group at a carbon atom

3.3. Five-membered heterocycles with a leaving group at a nitrogen atom

4. cine- and tele-Substitution in Nonaromatic Systems

5. Conclusions

References

\section{Introduction}

In 1998, F. Rose-Munch et al. summarized ${ }^{1}$ the achievements on indirect nucleophilic substitution in arenetricarbonylchromium and cationic arenetricarbonylmanganese complexes presenting several examples of cine- and tele-substitutions, as well the first example of cine- and tele-substitution in neutral cyclohexadienyltricarbonylmanganese complex (119 references). A long review, titled "Transition-MetalMediated Dearomatization Reactions" ${ }^{2}$, that appeared two years later only briefly mentioned cine- and telesubstitutions in arenetricarbonylchromium complexes, noting that both reactions probably involved the following sequence of reactions: nucleophilic addition, protonation, isomerization and elimination to reconstruct aromatic system (4 references). In 2001, we published the first general review article ${ }^{3}$ on the cineand tele-substitutions describing these types of reactions in homoarenes, heteroarenes and aliphatic systems, regardless of nucleophilic, electrophilic or radical mechanisms of the reactions (210 references). One year later, Rose-Munch and Rose published a microreview on cine and tele nucleophilic substitutions describing newer achievements for the reactions of (arene)tricarbonyl-chromium and tricarbonyl(n5cyclohexadienyl)manganese complexes (over 50 references). ${ }^{4}$ The same authors and Didier Astru reviewed ipso-, cine- and tele- nucleophilic aromatic substitutution of arenetricarbonylchromium complexes in a book chapter published in 2002 that became available online in $2004 .{ }^{5} \mathrm{~A}$ paper reviewing the progress in the radical reactions of arene metal complexes published in $2003^{6}$ reported no example of cine- or tele-substitutions. In their review of nucleophilic substitution of hydrogen in heterocyclic chemistry, Makosza and Wojciechowski ${ }^{7}$ devoted one short sub-chapter to cine- and tele-substitutions in heteroarenes (5 references). In their microreview, Peng and $\mathrm{Li}^{8}$ focused on the cine-substitution in Stille cross-coupling reactions which often occurred with sterically hindered vinyltin components (over 50 references). Much later Kaur et al. in a review ${ }^{9}$ on carbastannatranes as mediators in Stille coupling also gave an example of cine-substitution. Ni-catalyzed Suzuki-Miyaura, Fe-catalyzed Kumada cross-coupling reactions of phenol-based electrophiles and other reactions of unconventional phenol derivatives including cine-substituted products were reviewed by Mesganaw and Garg (5 references). ${ }^{10}$ In a review of the chemistry of nitroquinolones, Nishiwaki ${ }^{11}$ described the unusual reactivity of 1-methyl-3,6,8-trinitroquinolone resulting in cine-substitution of 3-nitro group (52 references). In 2011, Tishler ${ }^{12}$ presented a very comprehensive and interesting review of nucleophilic tele- 
substitution in various heteroaromatic systems (37 references). With the exception of a review by us ${ }^{3}$, none of these reviews tried to cover more or less the whole field of cine- and tele-reactions. The present paper is a continuation of the work ${ }^{3}$ published fifteen years ago. According to the IUPAC Gold Book definition ${ }^{13}$, cinesubstitution is a "substitution reaction (generally aromatic) in which the entering group takes up a position adjacent to that occupied by the leaving group". Similarly, the IUPAC Gold Book defined ${ }^{13}$ tele-substitution as a "substitution reaction in which the entering group takes up a position more than one atom away from the atom to which the leaving group was attached". Such terms as cine-addition, cine-cyclization, cine-elimination or cine-rearrangement, which also appear in the chemical literature, are not defined in the IUPAC Gold Book. cine- and tele-Substitutions probably are quite widespread but often they are not main processes and the terms cine or tele are not even mentioned in papers dealing with reactions of these types. Some authors consider such reactions as abnormal courses. In Web of Science, the term cine-substitution appeared in 63 papers published within the last fifteen years (Fig. 1) and 177 times since its introduction in 1968.
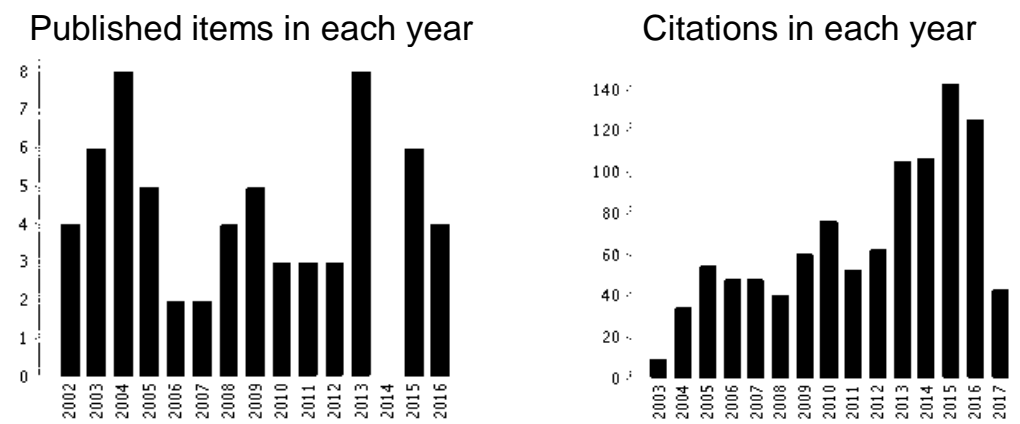

Figure 1. Number of papers using the term "cine-substitution" in the last fifteen years.

Often, these papers did not describe new cine-substitution reactions but merely cited the older papers. Most studies published within the recent fifteen years have been decidedly concentrated on the nucleophilic cine-substitution in conjugated systems such as homoarene complexes with metals or in heteroarenes. Most likely, only one paper dealt strictly with radical aromatic cine-substitution. Examples of cine- or telesubstitutions in aliphatic systems were reported very rarely as well. Studies of tele-substitution appears to be even less common recently (26 papers, Fig. 2) and have been mainly concerned with the reactions of heterocycles.

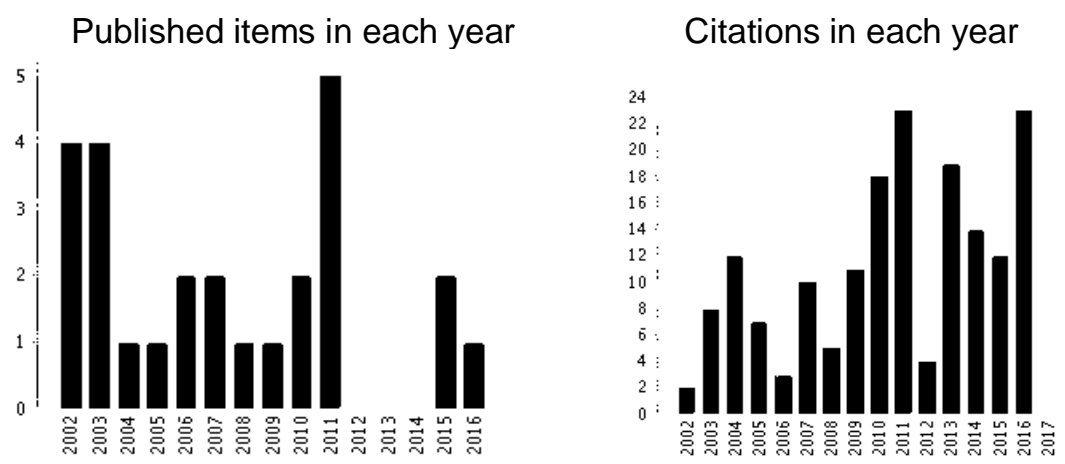

Figure 2. Number of papers using the term "tele-substitution" in the last fifteen years

Data taken from Web on Science on April 20, 2017. 
The term "tele-substitution" was introduced in 1977 by Tishler ${ }^{14}$ and, until 2016, was used in 68 papers reported by Web of Science.

\section{2. cine- and tele-Substitution of Homoarene Derivatives}

\subsection{Nitrohomoarenes}

In 2000, Giannopoulos et al. ${ }^{15}$ reported tele nucleophilic aromatic substitution of 1,3-dinitro-5trichloromethylbenzene with sodium methoxide with the formation of 4-methoxy-3,5-dinitrobenzaldehyde (55\%). Later, Garner et al. ${ }^{16}$ corrected that finding proving that 1,3-dinitro-5-trichloromethylbenzene (1) treated with sodium methoxide affords methyl 3,5-dinitrobenzoate (2, Scheme 1) and not 4-methoxy-3,5dinitrobenzaldehyde as was postulated earlier. ${ }^{15}$
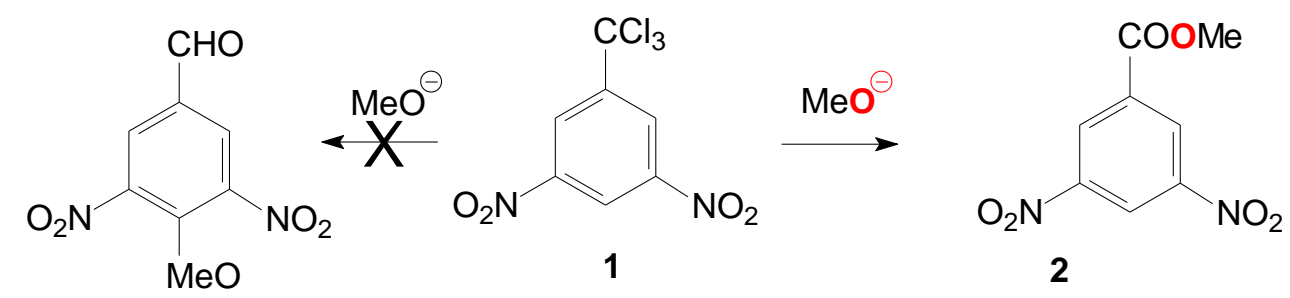

Conditions: $\mathrm{THF},-70{ }^{\circ} \mathrm{C}$, (ratio of arene to $\mathrm{MeMgCl}, 1: 1$ ), then $\mathrm{NH}_{3}($ liq. $),-70{ }^{\circ} \mathrm{C}$ to room temp.

Scheme 1. Reaction of 1,3-dinitro-5-trichloromethylbenzene with sodium methoxide.

Mąkosza et al. investigated oxidative aromatic nucleophilic substitution of hydrogen atom by Grignard reagents in nitrobenzenes containing chlorinated methyl group in the meta position. ${ }^{17}$ Grignard reagents attacked nitroarenes in ortho or para positions to form $\sigma^{H}$-complexes. In the absence of easy leaving groups, the complexes could then be oxidized to produce respective alkyl nitroarenes. No oxidizing reagent was necessary for the rearomatization of $\sigma^{H}$-complexes forming from 1-(chloro-, dichloro-, trichloromethyl)-3nitrobenzene with Grignard reagents. When 1-nitro-3-(trichloromethyl)benzene (3) was treated in tetrahydrofuran with methylmagnesium chloride at $-70{ }^{\circ} \mathrm{C}$ followed by the addition of liquid ammonia, a nucleophilic tele-substitution of chlorine atom by methyl group was observed, resulting in the formation of 3(dichloromethyl)-1-nitro-6-methylbenzene (4) in 21\% yield. cine-Substitution of compound 3 leading to product 5 was not observed (Scheme 2).

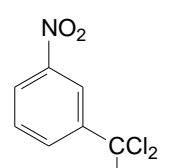

$3 \mathrm{Cl}$
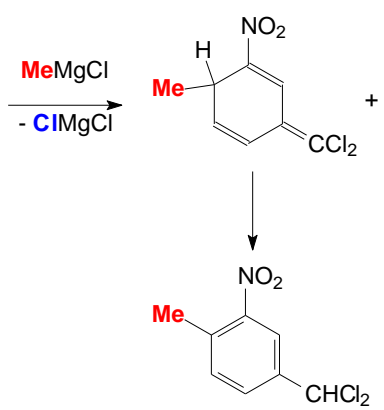

$421 \%$
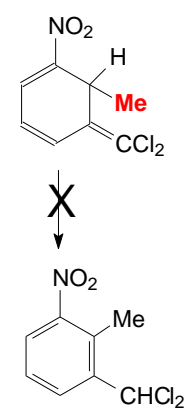

5

Scheme 2. tele-Substitution of 1-nitro-3-(trichloromethyl)benzene (3) with methylmagnesium chloride. 
Butylmagnesium chloride and 2-phenylethylchloride reacted with 1-nitro-3-(trichloromethyl)benzene (3) under similar conditions to give products of both substitution types: tele 6, 7 and cine 8, 9 (Scheme 3).

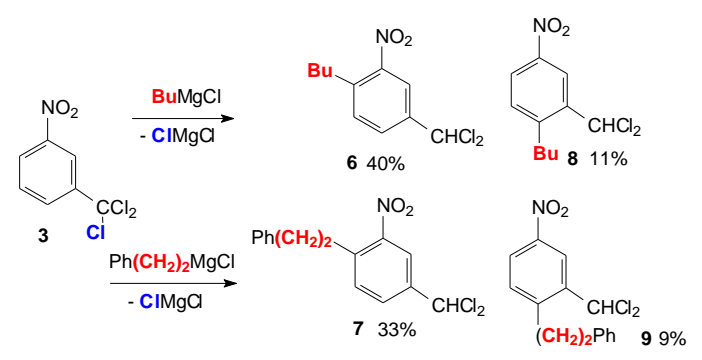

Scheme 3. cine- and tele-Substitution of compound $\mathbf{3}$ with butylmagnesium and benzylmagnesium chlorides.

1-Chloromethyl-3-nitrobenzene and 1-(dichloromethyl)-3-nitrobenzene treated with Grignard reagents according to the same procedure as compound $\mathbf{3}$ also afforded their respective tele-substituted products in moderate yields. In the course of the search for the reaction mechanism, it was found that alkylmagnesium chlorides used in equimolar amount added to the ortho or para positions with respect to the nitro group of nitrobenzene derivative substituted in position 3 with the chlorinated methyl group and formed their respective $\sigma^{H}$ adducts. The adducts released the chlorine anion from the chlorinated methyl group to give a cyclohexadiene intermediate which could be stabilized by 1,3- or 1,5-hydrogen shift following the addition of liquid ammonia to the reaction mixture. Nucleophilic tele-substitution was then observed. Liquid ammonia probably facilitated rearomatization. Cyclohexadiene intermediate in the presence of an excess of Grignard reagent reacted with a second molecule of the reagent to produce stable cyclohexene derivative.

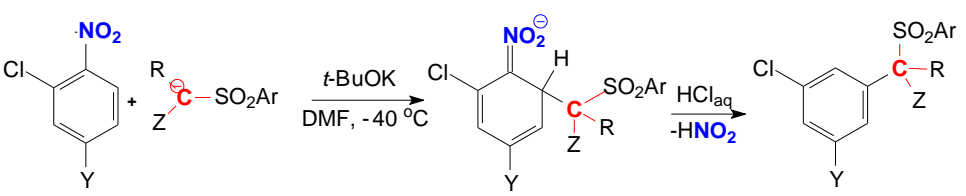

\begin{tabular}{|c|c|c|c|c|c|c|}
\hline \multirow{2}{*}{\multicolumn{2}{|c|}{$\begin{array}{r}10 a-c \\
\mathrm{ArNO}_{2} \\
\end{array}$}} & -15 & & \multicolumn{3}{|c|}{$11 a, 14 a, 15 a, 12 b, 13 b, 15 c$} \\
\hline & & \multicolumn{4}{|c|}{ Sulfone } & \multirow[t]{2}{*}{ Product* } \\
\hline No & $Y$ & No & $\mathrm{Ar}$ & $\mathrm{R}$ & Z & \\
\hline $10 a$ & $\mathrm{Cl}$ & 11 & $\mathrm{C}_{6} \mathrm{H}_{5}$ & $\mathrm{H}$ & $\mathrm{Cl}$ & $11 a$ \\
\hline $10 a$ & $\mathrm{Cl}$ & 12 & $\mathrm{C}_{6} \mathrm{H}_{5}$ & Me & $\mathrm{Cl}$ & $12 b$ \\
\hline $10 a$ & $\mathrm{Cl}$ & 13 & $\mathrm{C}_{6} \mathrm{H}_{5}$ & Et & $\mathrm{Cl}$ & $13 a$ \\
\hline $10 a$ & $\mathrm{Cl}$ & 14 & 4-Me- $\mathrm{C}_{6} \mathrm{H}_{4}$ & $\mathrm{H}$ & $\mathrm{C}_{6} \mathrm{H}_{5}$ & $14 a$ \\
\hline $10 a$ & $\mathrm{Cl}$ & 15 & 4-Me- $\mathrm{C}_{6} \mathrm{H}_{4}$ & $\mathrm{H}$ & $\mathrm{HC}=\mathrm{CH}_{2}$ & $15 a$ \\
\hline $10 \mathrm{~b}$ & $\mathrm{CF}_{3}$ & 13 & $\mathrm{C}_{6} \mathrm{H}_{5}$ & Et & $\mathrm{Cl}$ & $13 b$ \\
\hline $10 \mathrm{~b}$ & $\mathrm{CF}_{3}$ & 14 & 4-Me- $\mathrm{C}_{6} \mathrm{H}_{4}$ & $\mathrm{H}$ & $\mathrm{C}_{6} \mathrm{H}_{5}$ & $14 b$ \\
\hline $10 c$ & $\mathrm{CN}$ & 13 & $\mathrm{C}_{6} \mathrm{H}_{5}$ & Et & $\mathrm{Cl}$ & $13 c$ \\
\hline
\end{tabular}

*General reaction conditions: sulfone and nitroarene $(1: 1)$ in DMF is added at $-40{ }^{\circ} \mathrm{C}$ to a stirred t-BuOK (20\% exces to sulfone) solution in DMF and THF (12:1). After certain time the reaction is quenched with hydrochloric acid, the resulting mixture is diluted with water, extracted with dichloromethane and the extract is analysed chromatographycally.

Scheme 4. cine-Substitution of 2,4-disubstituted nitrobenzenes 10a-c with aryl alkyl sulfone carbanions 11-15. 
An ipso nucleophilic substitution of the chlorine atom in the chlorinated methyl group by a Grignard reagent was not observed. Makosza et al. ${ }^{18}$ also studied the behavior of 2,4-disubstituted nitroarenes with respect to the carbanions generated from alkyl aryl sulfones. Most important details concerning this new cinesubstitution reaction are shown in Scheme 4 with the accompanying table.

It was observed that the carbanions were added to the nitroarenes in the ortho position to the nitro group to form short lived $\sigma^{H}$ adducts. Protonation of the adducts led to the elimination of nitrous acid and formation of 1,3,5-trisubstituted benzenes as the products of a nucleophilic cine-substitution of the nitro group. Mąkosza et al. ${ }^{19}$ performed kinetic experiments for vicarious nucleophilic substitution of 2chloronitrobenzene, 4-chloro-nitrobenzene, 2,4-dichloronitrobenzene, 2,4,6-trichloronitrobenzene, 2bromonitrobenzene and 4-bromonitrobenzene by treating these compounds with chloromethyl phenyl sulfone in dimethylformamide in the presence of an excess of potassium $t$-butoxide at $-40{ }^{\circ} \mathrm{C}$. They measured the relative rates of formation of $\sigma$ adducts obtained by nucleophilic addition of carbanions to nitroarenes. In an experiment with 2,4-dichloronitrobenzene, the authors observed the formation of an unexpected product identified as chloro(3,5-dichlorophenyl)methyl phenyl sulfone (17), which is therefore a product of a new aromatic cine nucleophilic substitution (Scheme 5).

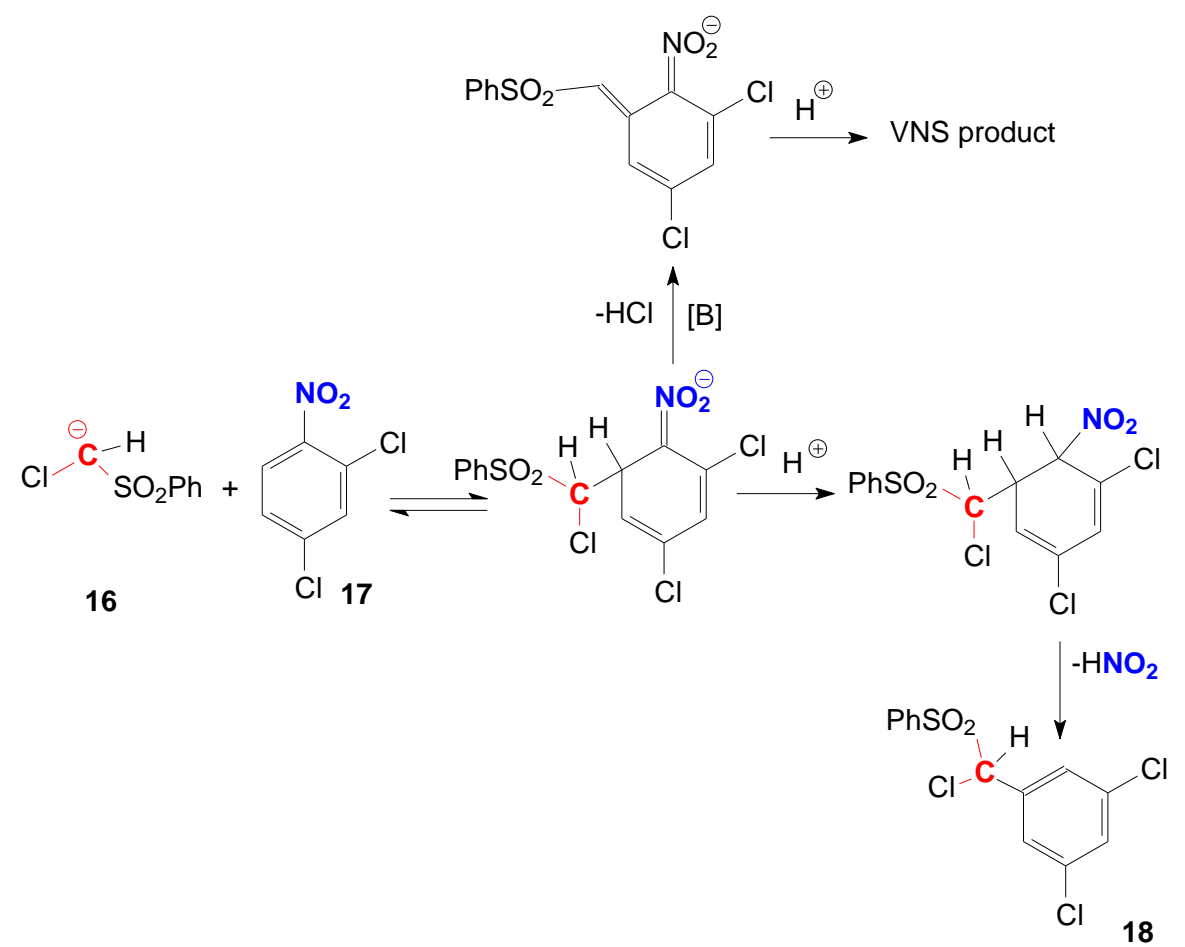

Scheme 5. Substitution of 2,4-dichloronitrobenzene (17) with chloromethyl phenyl sulfone carbanion (16) to produce cine-product 18.

In their conclusions, the authors stated that chlorine atoms activated nitroarene to attack the nucleophiles protecting the same positions that they occupied. In cooperation with Varounis, ${ }^{20}$ Mąkosza showed that the reaction of dialkyl malonates with 1-nitro-3-(trichloromethyl)benzene (3) led to mixtures of products 19-22 with the proportions depending mainly on the stoichiometry of the reactants. Then, they extended their former investigations of the tele-substitution in 1-nitro-3-(trichloromethyl)benzene (3) with Grignard reagents on 1-chloro-4-nitro-2-(trichloromethyl)benzene (23) and on carbanions derived from dialkyl malonates, 2-phenylacetonitrile and 2-phenylacetate (Scheme 6). 


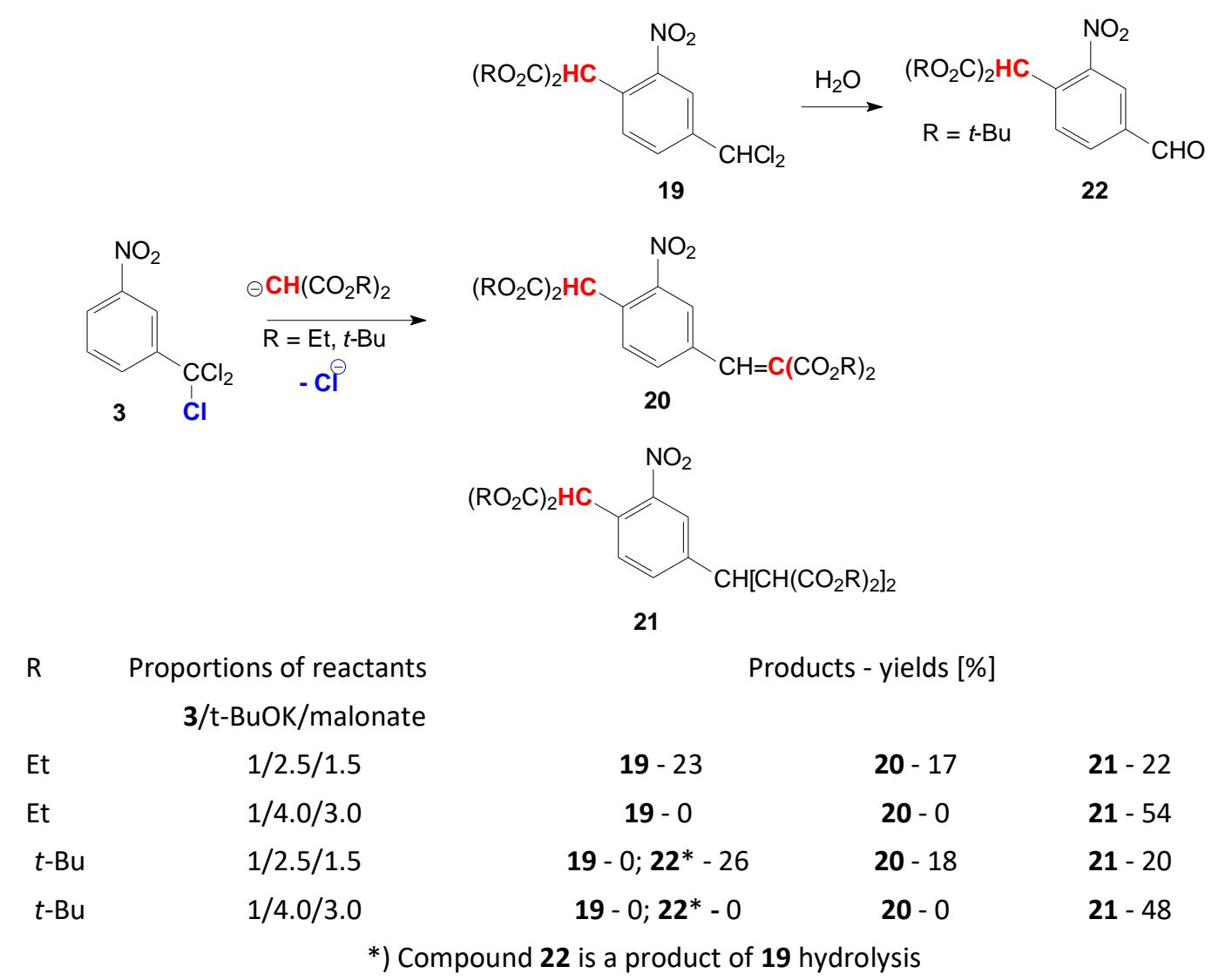

Scheme 6. Typical products of the tele-nucleophilic substitution of 1-nitro-3-(trichloromethyl)benzene (3) with dialkyl malonates.

A mechanism for the formation of compounds 19, 20 and 21 was postulated. The course of the reaction depended not only on the stoichiometry of reactants but also on the temperature of the reaction. Compound 3 or 1-chloro-4-nitro-2-(trichloromethyl)benzene (23) treated with diethyl malonate at $-30{ }^{\circ} \mathrm{C}$ afforded products $\mathbf{2 4}$ or $\mathbf{2 4}^{\prime}$ that were different than those obtained from the reaction at $0{ }^{\circ} \mathrm{C}$ (Scheme 7 ).

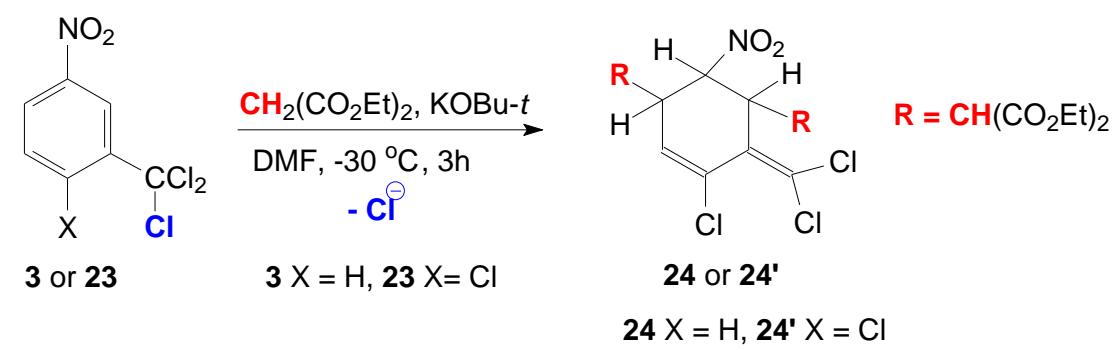

Scheme 7. Reaction of 1-nitro-3-(trichloromethyl)benzene (3) and 1-chloro-4-nitro-2-(trichloromethyl)benzene (23) with diethylmalonate at $-30^{\circ} \mathrm{C}$.

Conversion of substituted nitrobenzenes into benzoic acids under the action of an excess of potassium cyanide in aqueous ethanol is known as the von Richter reaction. It is an example of an aromatic nucleophilic cine-substitution. ${ }^{3}$ In the reaction, the carboxylic group replaces the hydrogen atom in the ortho-position to the leaving nitro group. The postulated ${ }^{21}$ mechanism of the von Richter reaction is shown in Scheme 8. 


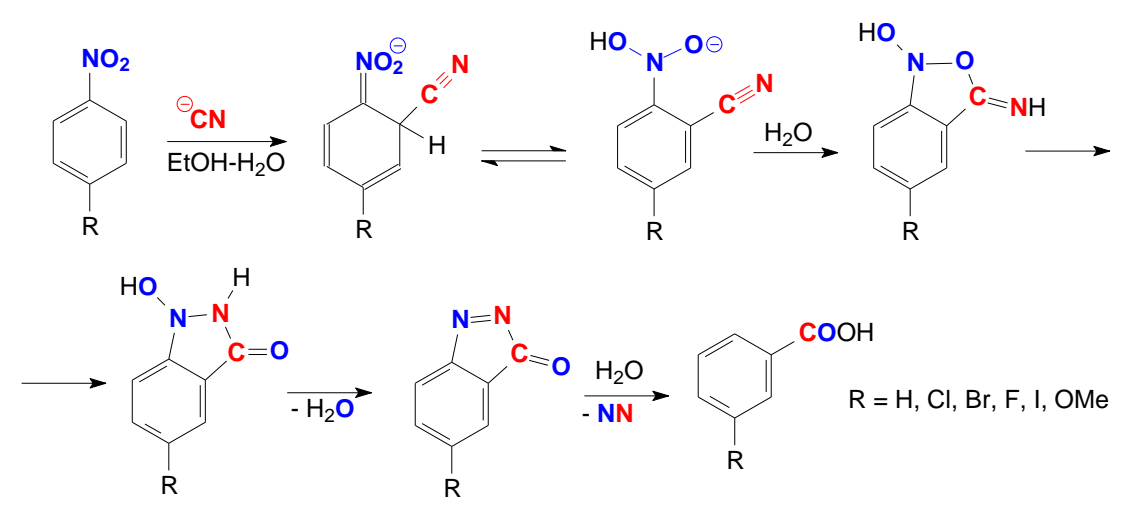

Scheme 8. Postulated mechanism of the von Richter reaction.

Makhova et al. $^{21}$ presented theirs results on the investigations of the first examples of ionic liquidpromoted von Richter reaction. They studied the conversion of electron-deficient para-substituted nitroarenes to the corresponding meta-substituted benzoic acids ${ }^{21}$ and found that the von Richter reaction of the investigated nitroarenes in the presence of ionic liquids (usually biimidazolium tetrafluoroborate) led to high yields of products including such compounds as 3-fluoro- or 3-methoxybenzoic acids which were practically not achievable under common reaction conditions. The authors also found that the von Richter reaction was slowed by electron-donating substituents in nitrobenzenes. The use of ionic liquids as solvents allowed to avoid or diminish the formation of tars in the reaction.

Miller et al. have recently published a paper ${ }^{22}$ in which they used the term "cine addition" for the chemical reduction of nitro to nitroso group in an aromatic compound (BZT) by the action of the thiolate anion (Scheme 9).

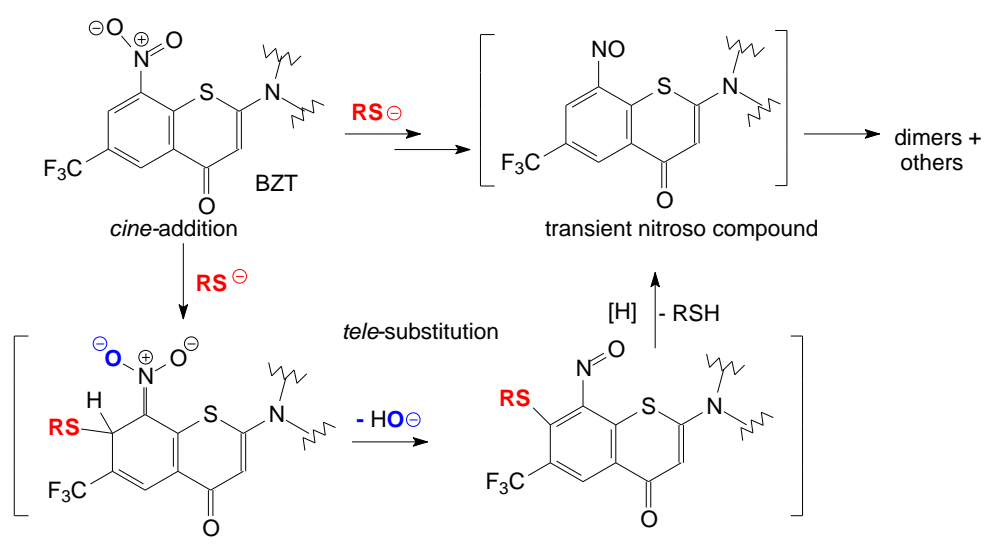

Scheme 9. Chemical activation (cine-addition of thiolate anion) of nitro group in BZT.

The term cine was used to indicate the location of an attack of the thiolate anion on the ortho position to the reduced nitro group in BZT. In light of the definitions of the terms cine and tele, we are of the opinion that a part of the reaction may also be treated as tele-substitution.

Kim et al. ${ }^{23}$ investigated oxidative nucleophilic substitution of hydrogen (ONSH) in 9-nitroanthracene (25) under the action of 2-naphthols and phloroglucinol. When compound 25 was treated with 2-naphthol in dimethylsulfoxide in the presence of $50 \%$ excess of cesium carbonate for seven hours at room temperature, the main unexpected product, namely, 1-(anthracen-9-yl)-2-naphthol (26,63\%), was formed as the result of 
tele substitution of the nitro group while the typical ONSH product 1-(10-nitroanthracen-9-yl)-2-naphthol (27) was obtained in $22 \%$ yield only (Scheme 10 ).

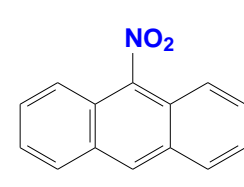

25

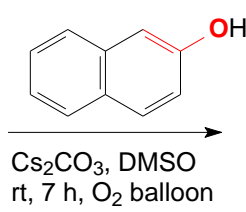

rt, $7 \mathrm{~h}, \mathrm{O}_{2}$ balloon

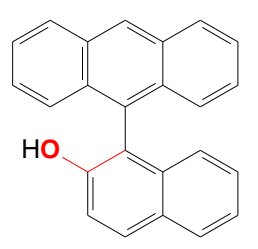

26, $63 \%$

tele-substitution

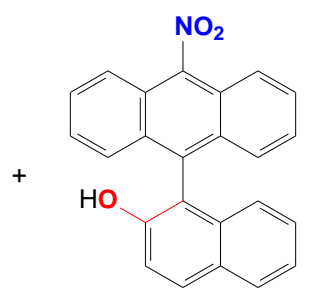

27, $22 \%$

ONHS product

Scheme 10. tele-Substitution of 9-nitroanthracene (25) with 2-naphthol.

Other 2-naphthols, namely, 2,7-dihydroxy-, 2-hydroxy-7-methoxy-, 2-hydroxy-8-methoxy- and 2hydroxy-3-methoxynaphthalene reacted in a similar way (Scheme 11).

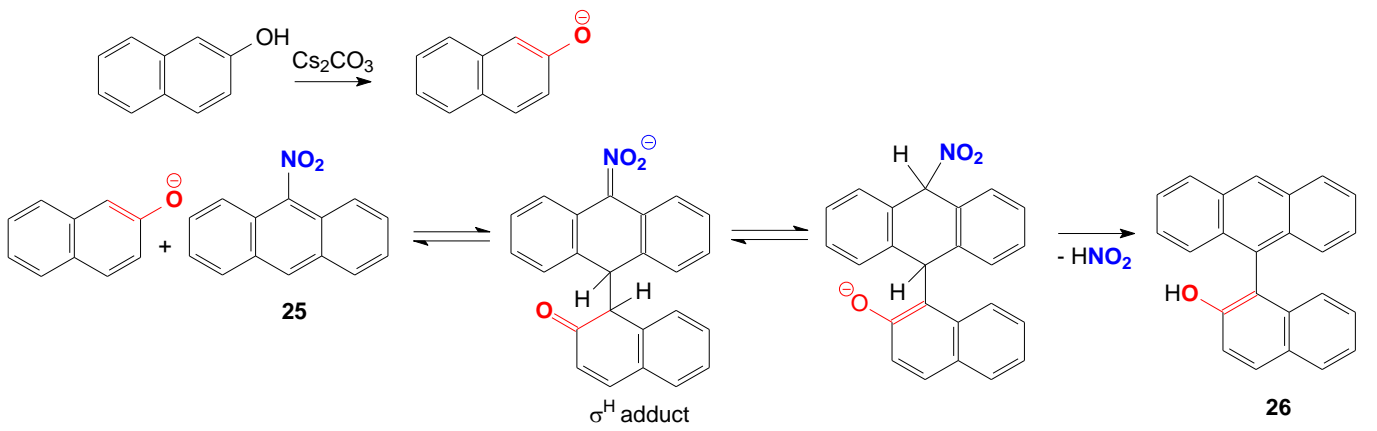

Scheme 11. Proposed mechanism of tele-substitution of 9-nitroanthracene (25) with 2-naphthol.

The yields of the tele substitution products exceeded the yields of the ONSH products for all naphthols. A mechanism of the reaction was postulated. Nitroarene $\mathbf{2 5}$ under the action of the more nucleophilic phloroglucinol gave two tele-substitution products (Scheme 12).
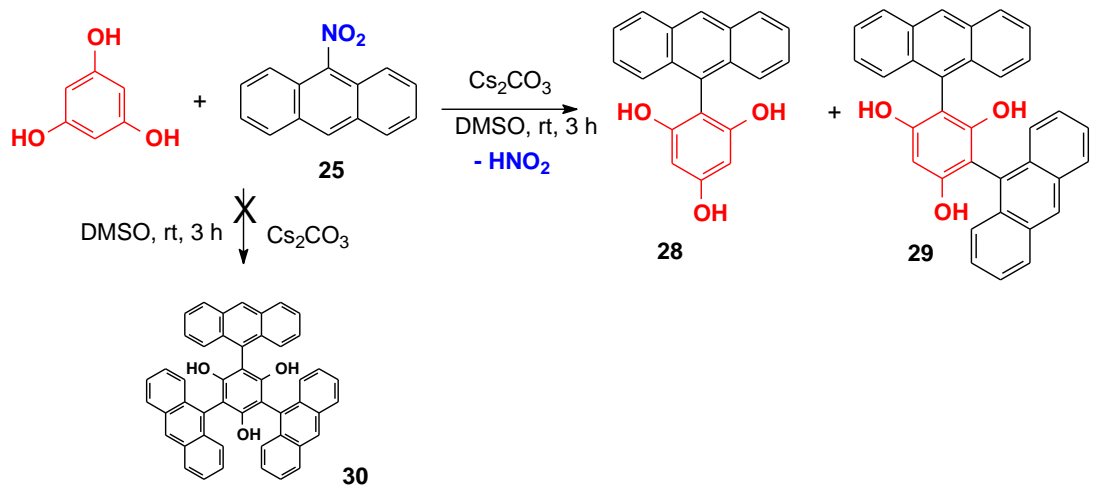

Scheme 12. tele-Substitution of 9-nitroanthracene (25) with phloroglucinol. 
The previously formed tele-product 2-(anthracen-9-yl)phloroglucinol (28) reacted with the second molecule of compound 25 to give 2,4-di(anthracen-9-yl)phloroglucinol (29). The proportions of these two products depended mainly on the proportions of the reactants. Formation of 2,4,6-tri(anthracen-9$\mathrm{yl}$ )phloroglucinol (30) was not observed. A nucleophilic aromatic predominating tele-substitution was also typical for the reaction of nitroarene 25 with 1,3-cyclohexanediones $\mathbf{3 1}$ (Scheme 13).

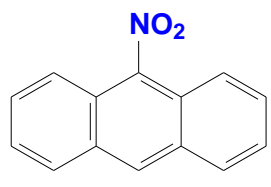

25
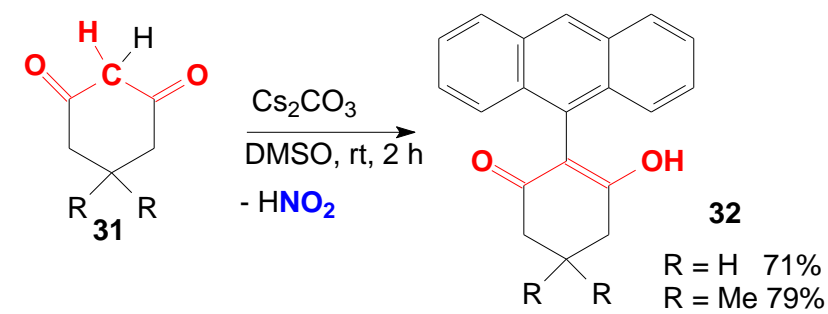

Scheme 13. Reaction of 9-nitroanthracene (25) with 1,3-cyclohexanediones 31; the formation of telesubstitution products 32 .

\subsection{Complexes of homoarenes with metals}

As mentioned in the introduction, three reviews have dealt with cine-substitution of homoarene complexes with metals, but nevertheless some new papers should be presented here. A review of radical aromatic substitution of arenetricarbonylchromium complexes published in $2003^{6}$ did not even mention the terms cine or tele even though one year earlier Li et al. ${ }^{24}$ presented the results of their investigations of radical aromatic substitution of $\left(\eta^{6}\right.$-chloroarene)tricarbonylchromium complexes $\mathbf{3 3}$. When complexes 33 were treated with acetone and samarium iodide in a tetrahydrofuran/hexamethylphosphoramide solution containing $t$-butanol as the proton source, cine- and tele-substitution products were formed. Other ketones and aldehydes e.g., cyclopentanone, cyclohexanone, propanal or butanal reacted accordingly (Scheme 14).

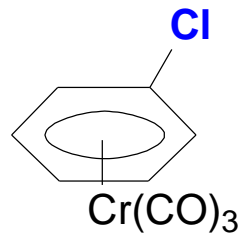

33

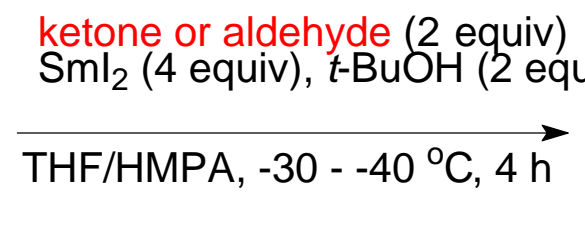

ketone or aldehyde (2 equiv)

THF/HMPA, $-30--40{ }^{\circ} \mathrm{C}, 4 \mathrm{~h}$ 


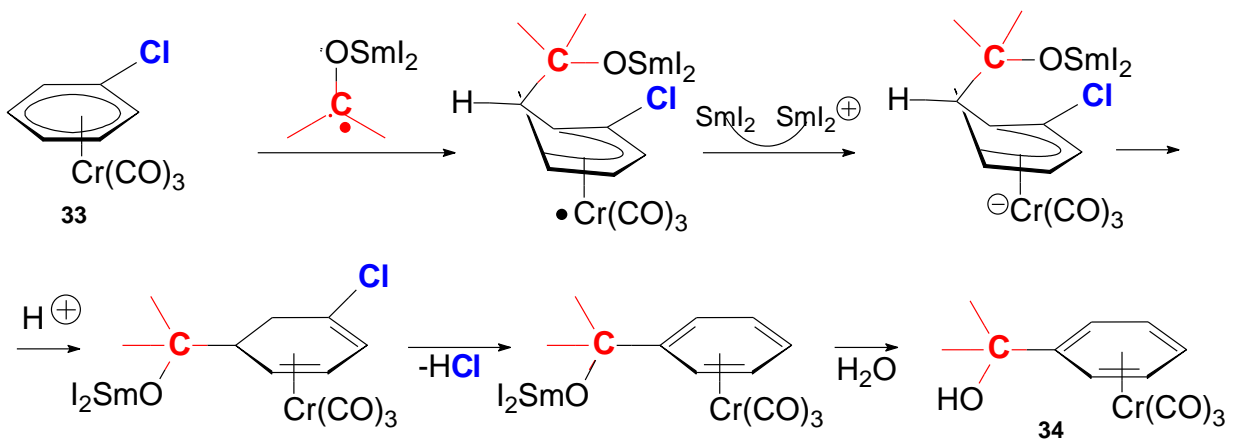

Scheme 15. Proposed mechanism of meta-tele-substitution of chlorine atom by a carbonyl compound in $\left(\eta^{6}-\right.$ chloroarene)tricarbonylchromium complex (33) in the presence of samarium iodide.

Xia et al. ${ }^{25}$ investigated the cine-substitution of metallabenzenes both experimentally and by density functional theory (DFT) quantum mechanical calculations. The authors presented the first examples of the cine-substitution reactions of metalla-aromatic compounds showing that alkali-resistant osmabenzene complex 35 reacted with alcohols in the presence of sodium alkoxides to afford exclusively cine-substituted products 36 (Scheme 16).

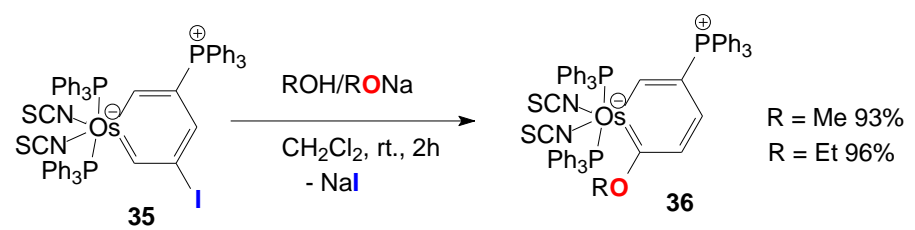

Scheme 16. cine-Substitution of iodine atom by alkoxy group in osmabenzene complex $\mathbf{3 5 .}$

In contrast to that, complex $\mathbf{3 5}$ treated with amines in the presence of e.g., sodium methoxide gave five-membered ring compounds $\mathbf{3 7}$ with cine-substitution as a side reaction only. The authors assumed that strong alkalis did not take part in the reaction with amines. A mechanism of the reactions of osmabenzene complex 35 with nucleophiles was postulated. DFT calculations supported the experimental findings concerning the course of the reactions. Conclusions of NMR in situ and from labeled compounds also appeared to support the proposal that the studied cine-substitution reactions occurred in three following key steps: addition of a nucleophile-ring opening-ring closure, therefore according to an ANRORC mechanism (Scheme 17).

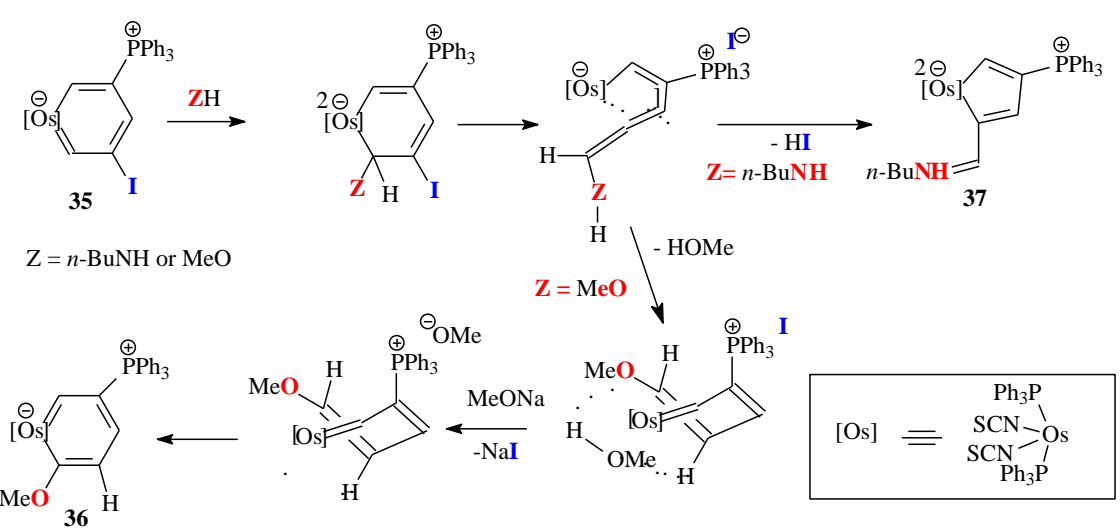

Scheme 17. Postulated mechanism of reactions of osmabenzene complex 35 with nucleophiles (alkoxides or amines). 
cine-Substitution accompanied by the formation of ipso-substitution (40) of aryl bromides (39) in SuzukiMiyaura coupling reaction catalyzed by dinuclear Pd complexes (38) was reported (Scheme 18, product 41). ${ }^{26}$

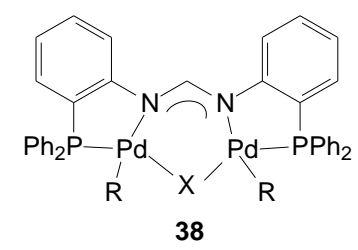

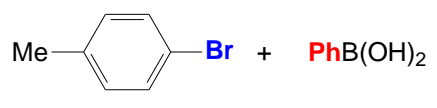

39

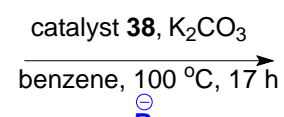

$-\mathrm{Br}$

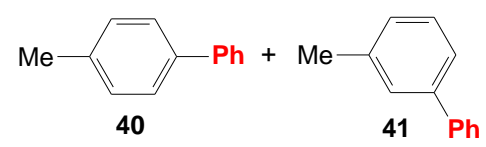

Scheme 18. ipso- (40) and cine-Substituted (41) products from $p$-bromotoluene (39) in Suzuki-Miyaura crosscoupling catalyzed by dinuclear palladium complex 38 where: $\mathrm{R}=p-\mathrm{MeC}_{6} \mathrm{H}_{4}, p-\mathrm{ClC}_{6} \mathrm{H}_{4}$ or $\mathrm{Me}$ and $\mathrm{X}=\mathrm{OH}, \mathrm{Br}, \mathrm{Cl}$ or I.

The highest proportion (ca. 30\%) of cine-substituted $\mathbf{4 1}$ to ipso-substituted product $\mathbf{4 0}$ was obtained with the use of complex 38 in which $\mathrm{R}$ was $p-\mathrm{MeC}_{6} \mathrm{H}_{4}$ and $\mathrm{X}$ was I. In addition to compound 39, various other monosubstituted bromobenzenes were treated with phenylboronic acid in the presence of catalyst 38 . In most of the reactions, the yields of cine-substituted compounds did not exceed $25 \%$ and bromoarenes were still present in the post-reaction mixtures. Ratios of cine- to ipso-substituted products did not depend on para or meta position of substituents and their electronic properties. tele-Substituted (ortho) products were not observed at all. By contrast, the yields of cine-substituted products varied with the types of the orthosubstituents in the case of starting ortho-substituted bromobenzenes. The effect of the solvent on the ratios of cine- to ipso-substitution was also studied, showing that benzene strongly (ca. $4: 1$ ) favored ipsosubstitution. When the reaction was performed in per-deuterated benzene, the ipso-position in the cinesubstituted product was also deuterated to a great extent. The deuteration did not occur in the reaction catalyzed by a mononuclear palladium complex e.g., [ $\left.\mathrm{Pd}\left(\mathrm{PPh}_{3}\right)_{4}\right]$. A dinuclear $\mathrm{Pd}$ complex appeared to be necessary also for cine-substitution because mononuclear $\mathrm{Pd}$ complex was proved not to be active in this case. The mechanism of the reaction is still not fully clear. As shown in Scheme 19, the ipso-position of metamethoxybiphenyl (43) was highly deuterated.
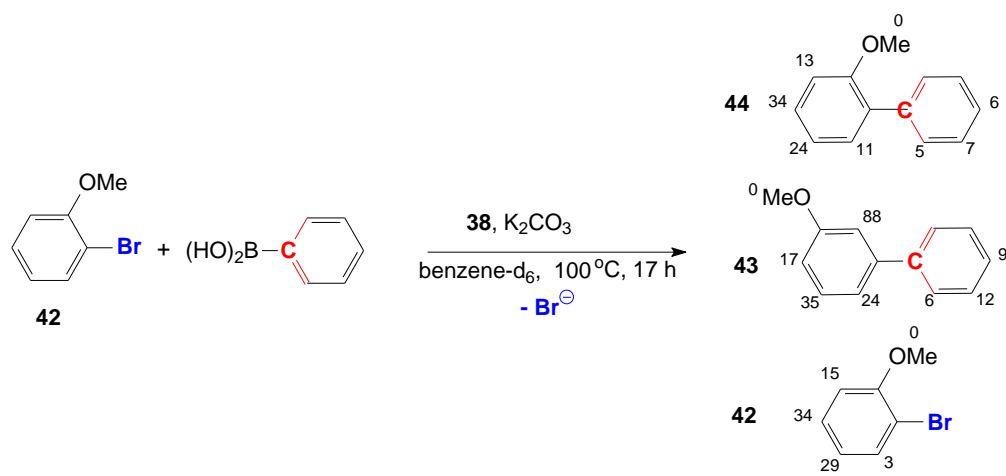

Scheme 19. Distribution of deuterium in products of reaction of ortho-bromoanisole (42) in benzene- $d_{6}$; the smaller numbers close to structural formulas indicate the percentage of deuteration in a given position. 
The ipso-H-atom was most likely derived from the solvent. Unexpectedly, H/D exchange reactions occurred in various positions of the benzene rings in meta- (43) and ortho-methoxybiphenyl (44) as well as in recovered ortho-bromoanisole (42). The reaction with benzene- $\mathrm{d}_{6}$ may indicate a mechanism of the reaction via the respective aryne as is depicted in Scheme 20.

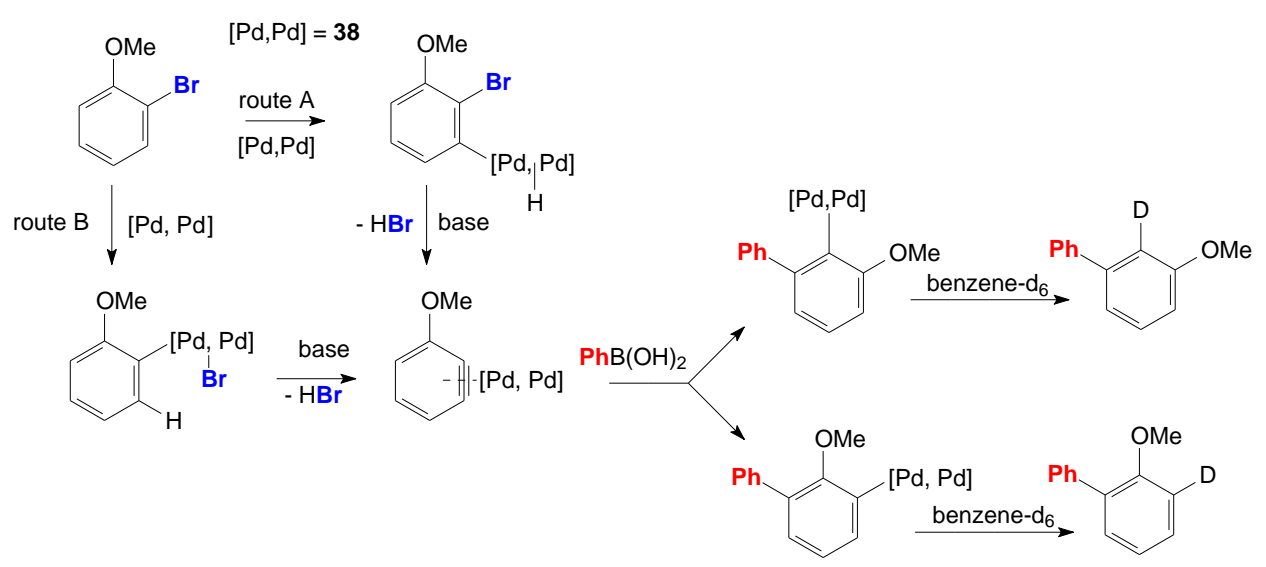

Scheme 20. The proposed mechanism of Suzuki-Miyaura cross-coupling of ortho-bromoanisole withphenylboronic acid in the presence of dinuclear palladium complex in perdeuterated benzene.

\subsection{Other homoarene derivatives}

Ohno at al. ${ }^{27,28}$ studied the behavior of $N$-(2-iodophenyl)- $N$-methylbenzamides toward samarium(II) iodide. Compound $45\left(R^{1}=H, R^{2}=M e\right)$ treated with samarium(II) iodide in the absence of the proton donor gave dimethylphenanthridone $\mathbf{4 6}$ as the predominant product. By contrast, benzanilide derivative $\mathbf{4 5}\left(R^{1}=M e, R^{2}\right.$ $=\mathrm{H}$ ) treated also with samarium(II) iodide but in the presence of isopropanol as a proton source underwent spirocyclisation to afford diene $\mathbf{4 7}$ in an excellent yield (Scheme 21).
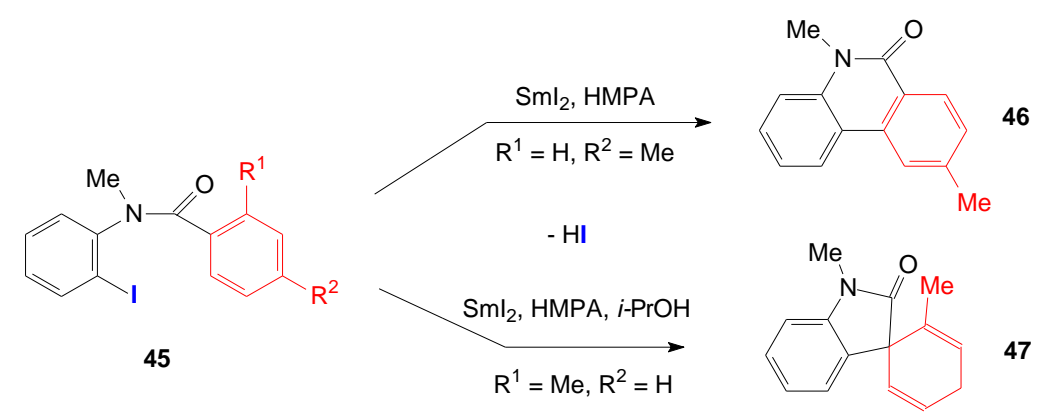

Scheme 21. Cyclization of ortho-iodobenzanilides 45 treated with samarium(II) iodide.

Product $\mathbf{4 6}$ was possibly formed via the rearrangement of the spirocyclohexadienyl radical. The best yields of spiroproducts were obtained from ortho-substituted benzanilide derivatives e.g., from compound $\mathbf{4 8}$. The ortho-substituents hindered the rearrangement and the intermediate radicals become sufficiently stable to be reduced and then protonated to afford spiroproduct $\mathbf{4 9}$ in high yield while the cine-cyclized phenanthridone derivative $\mathbf{5 0}$ can be a by-product only (Scheme 22). 


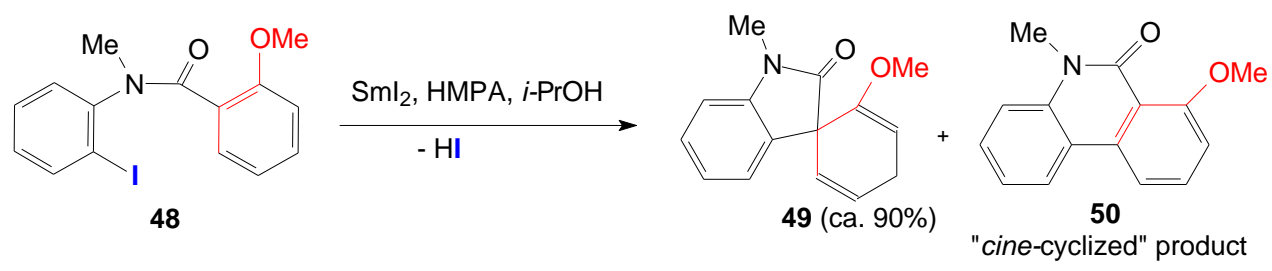

Scheme 22. Effect of ortho-methoxy group in iodobenzanilide $\mathbf{4 8}$ on the ratio of spirocyclized $\mathbf{4 9}$ and "cinecyclized" 50 product.

Therefore, the studies showed that intramolecular arylation of aryl radicals mediated by $\mathrm{Sml}_{2} / \mathrm{HMPA}$ in the presence of $i$-PrOH led to spirocycles and to reduced "cine-cyclized compounds". The spirocyclization proceeded via a cyclohexadienyl anion intermediate and 1,4-reduction of the cyclohexadiene intermediate. In most cases, spirocyclization was difficult and cine-cyclized products were formed in considerable amounts. Reactions of indole derivatives were an exception to this rule. Three possible mechanisms of cyclization of a radical derived from e.g., ortho-iodoanilides in the presence of samarium(II) iodide are shown in Scheme 23.

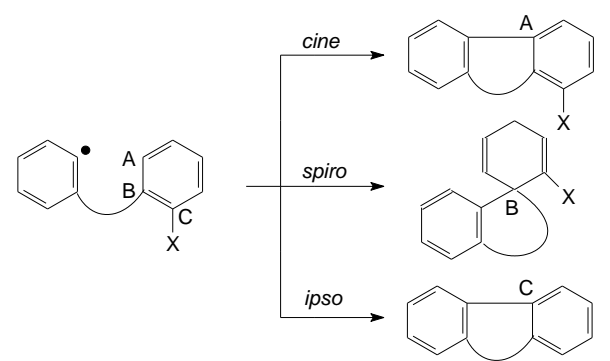

Scheme 23. Three possible ways of cyclization of a radical derived from e.g. ortho-iodoanilides in the presence of samarium(II) iodide.

A postulated mechanism of cine-cyclization of ortho-iodobenzanilides mediated by samarium(II) iodide is depicted in Scheme 24.

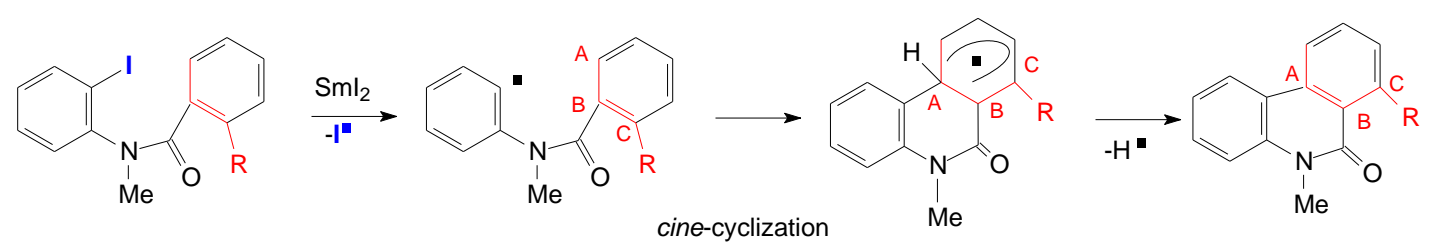

Scheme 24. Radical mechanism of cine-cyclization of iodobenzanilides to phenanthridones.

Mesganaw et al. ${ }^{29}$ elaborated and reported an efficient method of cine-substitution of various arenes with the methodology based on the use of aryl $O$-carbamates as an easily removable directing group. The idea of cine-substitution of arenes via aryl $O$-carbamates is presented in Scheme 25.

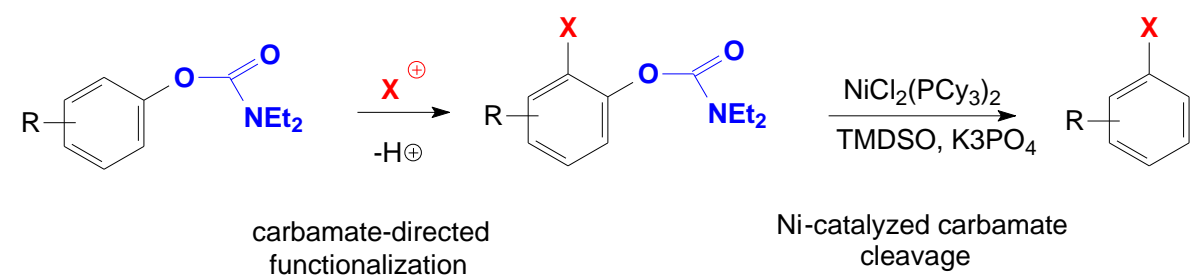

Scheme 25. An idea of cine-substitution of aryl $\mathrm{O}$-carbamates with $\mathrm{Ni}$-catalyzed carbamate cleavage. 
Very recently Tobisu and Chatani in a review ${ }^{30}$ on nickel-catalyzed cross-coupling reactions have cited work by Mesganaw giving an example of the cine-substitution. cine-Substitution based on the use of aryl $O$ carbamates as readily removable directing groups for arene functionalization provided a new tool for the preparation of some important arene and heteroarene derivatives. A key part of this tool is the reductive cleavage of aryl carbamates. The cleavage proceeded well under the following reaction conditions: homo- or heteroaryl carbamate (1 equiv), $\mathrm{NiCl}_{2}\left(\mathrm{PCy}_{3}\right)_{2}$ (5-20 mol \%), TMDSO (2.5 equiv), $\mathrm{K}_{3} \mathrm{PO}_{4}$ (4.5 equiv), toluene (0.27 M), $115-125^{\circ} \mathrm{C}$ for $15 \mathrm{~h}$. Examples of Mesganaw's method applications are depicted in schemes 26, 27 and 28.

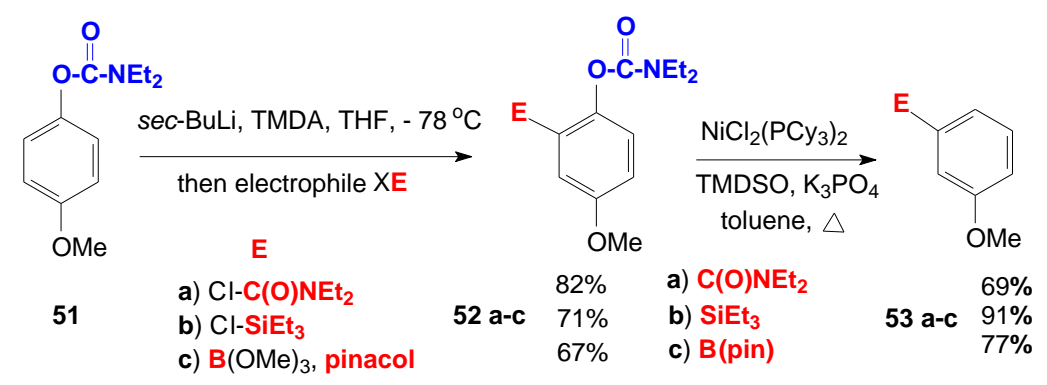

Scheme 26. Examples of cine-substitution of aryl $O$-carbamates.

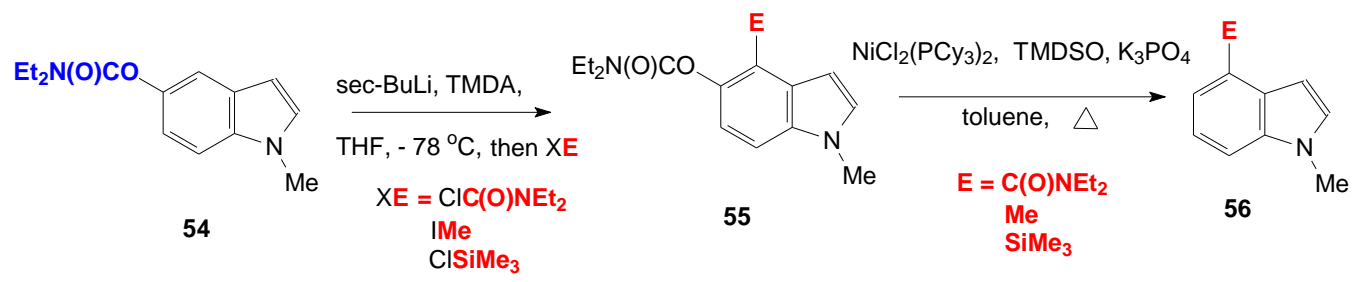

Scheme 27. Examples of cine-substitution of indolylcarbamate 54.

$\mathrm{Et}_{2} \mathrm{~N}(\mathrm{O}) \mathrm{CO}$

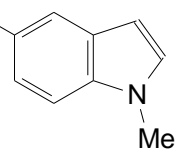

54

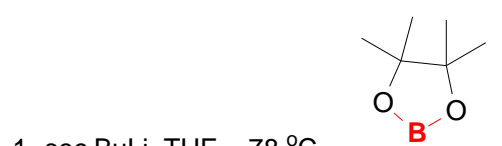

1. sec-BuLi, THF, $-78^{\circ} \mathrm{C}$

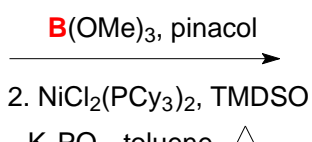

$\mathrm{K}_{3} \mathrm{PO}_{4}$, toluene, $\triangle$

(2 steps, 62\%)

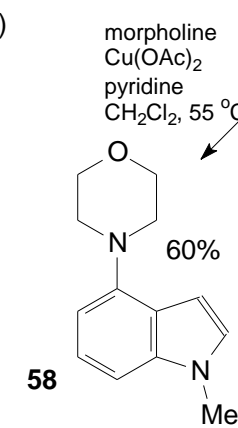

borylation with

cine-substitution

57
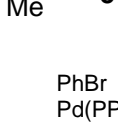

$\mathrm{Pd}\left(\mathrm{PPh}_{3}\right)_{4}$ $\mathrm{K}_{3} \mathrm{PO}_{4}$ toluene, $90^{\circ} \mathrm{C}$

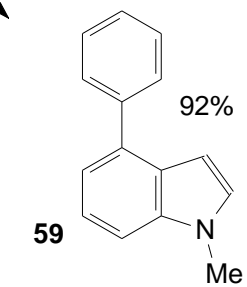

Scheme 28. Examples of cross-coupling approaches to cine-substituted products from $N$-methylindolylcarbamate 54. 
Very recently, a paper has been published ${ }^{31}$ proving that arylzinc reagents bearing electron-donating substituents were able to undergo cine-substitution with certain electrophiles such as perpivaloylated glucoside bromide or benzohydryl bromide under the conditions where stereoelectronic factors promoted cine- versus ipso-substitution (Scheme 29).

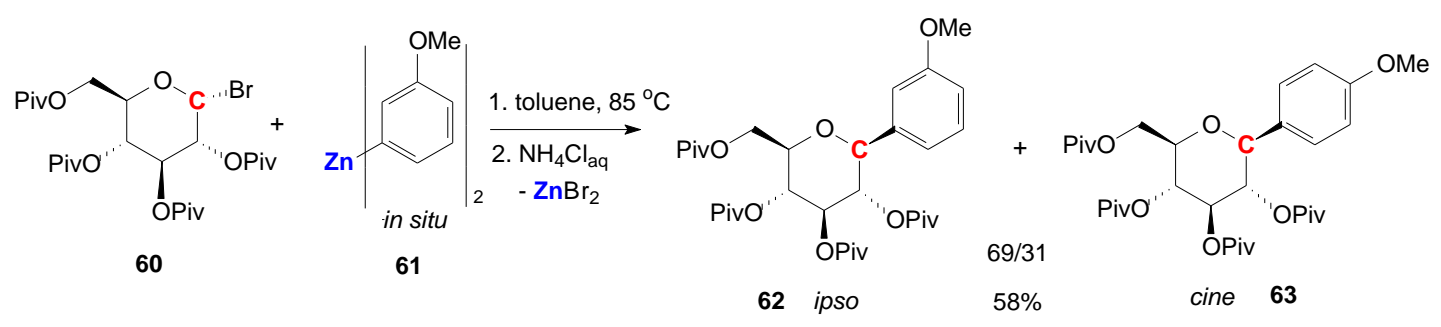

Scheme 29. Substitution (ipso versus cine) of meta-anisylzinc reagent with perpivaloylated glucoside.

A mechanism of the substitution was studied and proposed. It was established that cine- and telesubstitutions occurred according to the Friedel-Crafts type mechanism consisting of two competing intramolecular pathways. In one of the pathways, the carbon-zinc bond remained untouched. An example of cine- and tele-substitution reactions of benzhydryl bromide using arylzinc reagents is shown in Scheme 30.

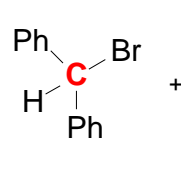

64

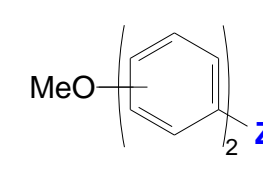

65

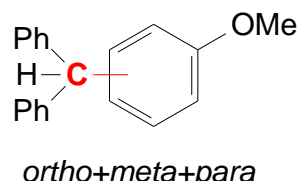

66

Distribution of product 66

$\begin{array}{cccc}\text { diarylzinc } 65 & \text { ortho } & \text { meta } & \text { para } \\ \text { ortho } & \text { ipso 38\% } & \text { cine 0\% } & \text { tele 62\% } \\ \text { meta } & \text { cine } 9 \% & \text { ipso 6\% } & \text { cine } 85 \% \\ \text { para } & \text { tele } 8 \% & \text { cine } 0 \% & \text { ipso 92\% }\end{array}$

Scheme 30. Substitution (cine, tele, ipso) of benzhydryl bromide 64 with anisylzinc reagents 65 .

\section{Nucleophilic cine- and tele-Substitution of Heteroarene Derivatives}

\subsection{Six-membered heterocycles with a leaving group at a carbon atom}

Nishiwaki et al. ${ }^{32}$ proposed a new method for the functionalization of quinolones. When 1-methyl-3,6,8trinitro-2-quinolone 67 was reacted with 1,3-dicarbonylcompounds in the presence of trimethylamine, a regioselective reaction occurred to afford cine-substitution product 68 forming according to the typical addition-elimination mechanism (Scheme 31). 


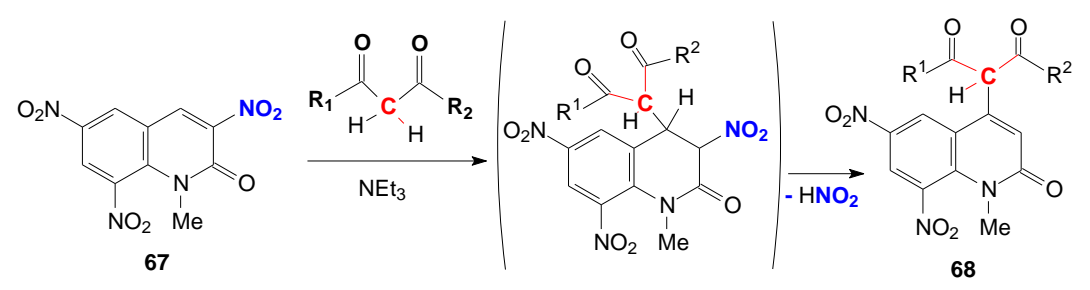

Scheme 31. cine-Substitution of trinitroquinolone 67 with carbanion derived from a 1,3-dicarbonyl compound.

Based on this finding, the authors elaborated a method for a simple preparation of 1-methyl-6,8dinitro-2-qinolone by treating compound $\mathbf{6 7}$ with tertiary or quaternary amines which contained at least two long alkyl chains. Chupakhin et al. ${ }^{33}$ elaborated a new procedure for the synthesis of some 1,2,4-triazine derivatives with chlorinated methyl groups at the position 3. One type of such derivatives, namely, 6-aryl-3trichloromethyl-1,2,4-triazines (69) reacting with indoles (70) or phenols (71) afforded tele-substituted products 6-aryl-5-indolyl- or 6-aryl-5-hydroxyphenyl-3-dichloromethyl-1,2,4-triazines (72), respectively (Scheme 32). This example of tele-substitution was mentioned in a review by Kovalev et al. on the nucleophilic substitution of hydrogen in arenes and hetarenes ${ }^{34}$.

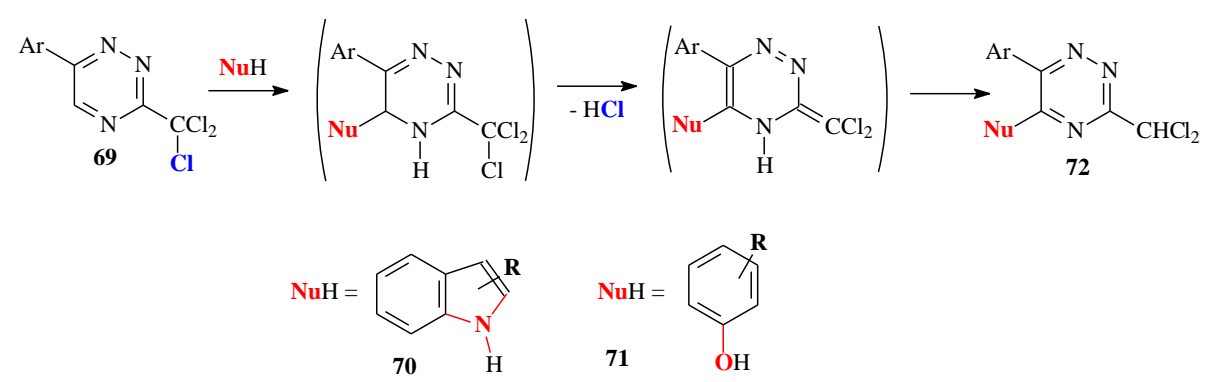

Scheme 32. tele-Substitution of triazines 69 with indols 70 or phenols 71 to afford new derivatives 72.

Yields of tele-products (72) exceeded $50 \%$. The reaction of dichloropyrazines $\mathbf{7 3}$ and $\mathbf{7 5}$ with a dithiane anion was described by Torr et al. ${ }^{35}$ The reaction (after the decomposition of dithiane protecting group with methyl iodide) produced the expected isomeric formylated chloropyrazines $\mathbf{7 4}$ and $\mathbf{7 6}$ respectively (Scheme 33).

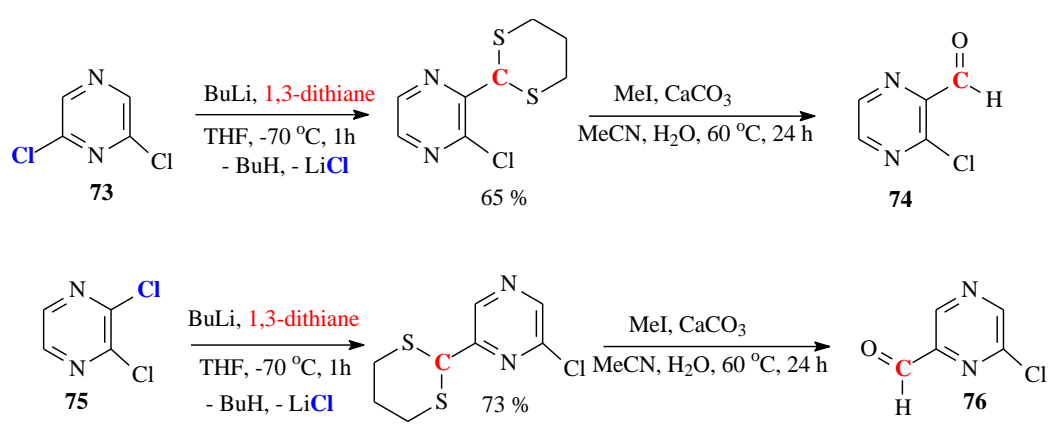

Scheme 33. Indirect tele-formylation of dichloropyrazines $\mathbf{7 3}$ and $\mathbf{7 5 .}$ 
A tele-substitution mechanism was studied using deuterium derivatives and was postulated by taking into considerations all the experimental observations (Scheme 34). A formation of cine-substitution product was not observed.
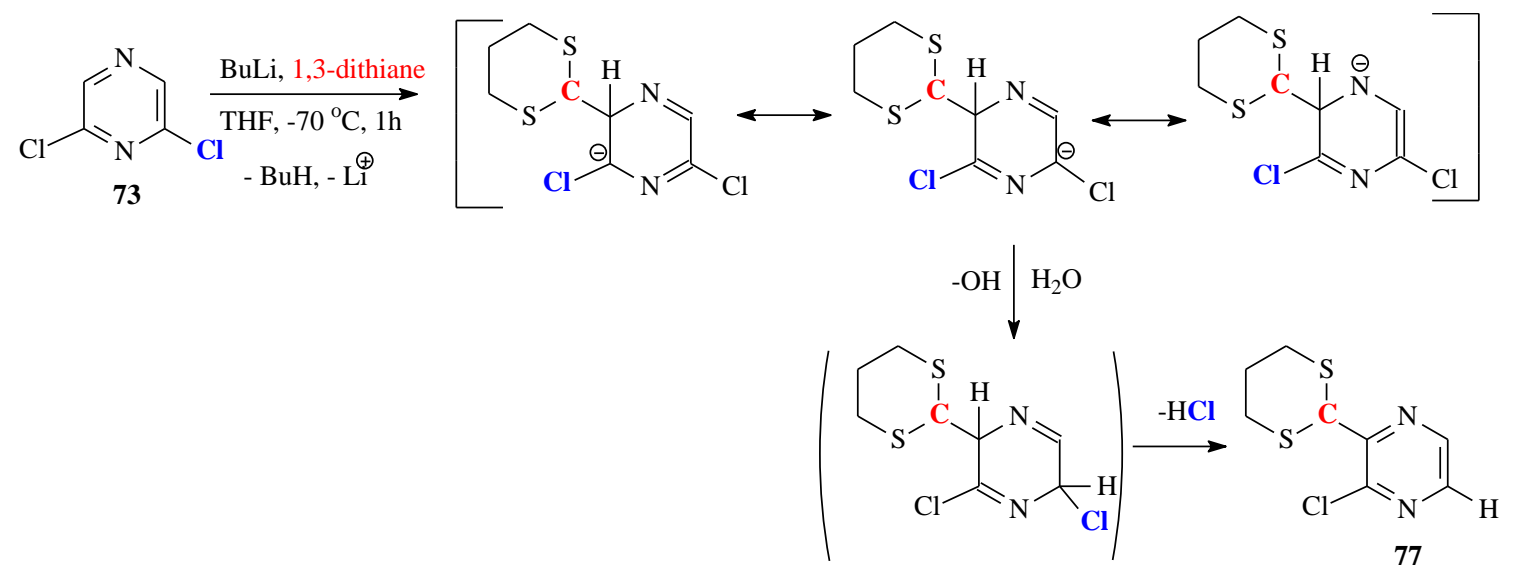

Scheme 34. A postulated mechanism of tele-substitution of dichloropyrazine $\mathbf{7 3}$ with lithiated dithiane to give compound 77.

In contrast to the reaction with the dithiane anion, dichloropyrazines $\mathbf{7 3}$ and $\mathbf{7 5}$ were ipso-substituted with morpholine. Pfleiderer et al. ${ }^{36}$ treated 1,3-dimethylpteridine-7-styryl-2,4(1H,3H)-dione (78a) or 1,3-dimethylpteridine-7-[2-(methoxycarbonyl)ethenyl-2,4(1H,3H)-dione (78b) with bromine and then with nucleophiles to give tele-substituted products 80 (Scheme 35 ).

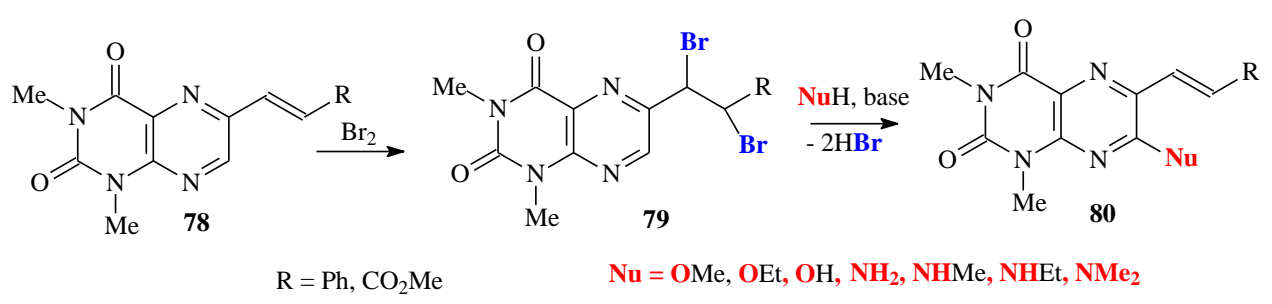

Scheme 35. Nucleophilic tele-substitution of product of bromine addition to pteridine derivatives 78.

1,3-Dimethylpteridine-6-[2-(methoxycarbonyl)ethenyl-2,4(1H,3H)-dione under action of bromine and then a nucleophile behaved differently, not giving the tele-substituted products. Enamines $\mathbf{8 1}$ were added to synthetic 1-methyl-3,6,8-trinitro-2-quinolone (67) and products of the addition were then hydrolyzed to furnish 4-acylmethyl-1-methyl-6,8-dinitro-2-quinolones (82, Scheme 36). ${ }^{37}$ 


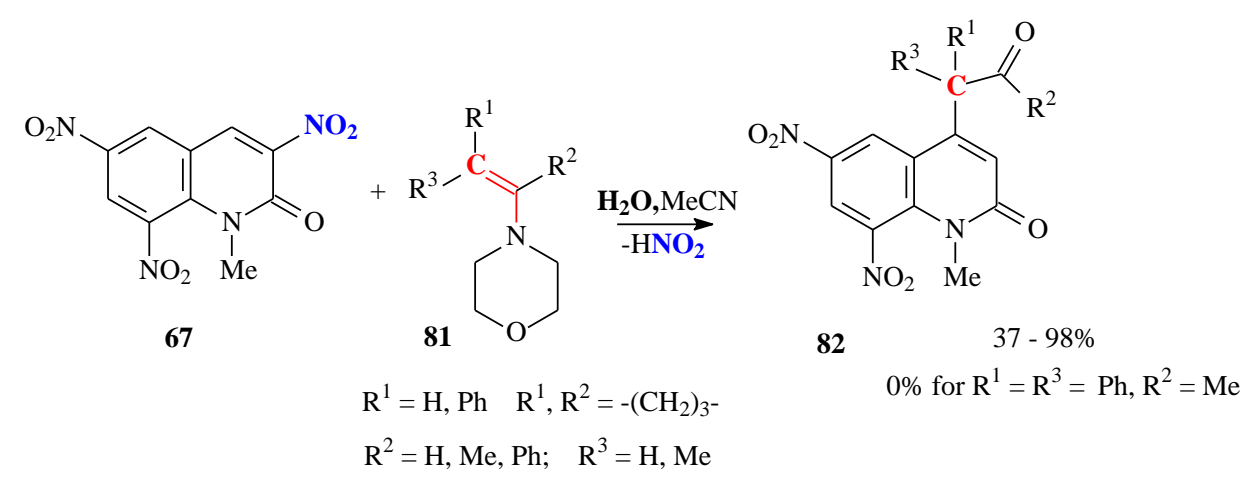

Scheme 36. cine-Substitution of trinitroquinolone 67 with enamines 81.

Compounds 82 were also obtained in reaction of quinolone 67 with certain ketones in the presence of triethylamine. A plausible mechanism of cine-substitution of the nitro group in compound 67 by the acylmethyl group present in products $\mathbf{8 2}$ was formulated (Scheme 37 ).

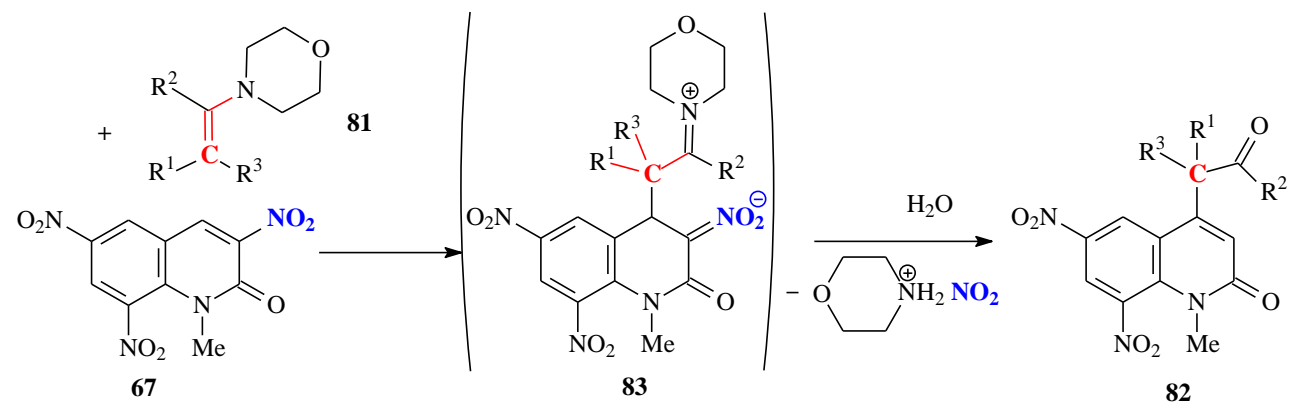

$\mathrm{R}^{1}=\mathrm{H}, \mathrm{Ph}, \quad \mathrm{R}^{2}=\mathrm{Me}, \mathrm{Ph} ; \quad \mathrm{R}^{3}=\mathrm{H}, \mathrm{Me}$

Scheme 37. Mechanism of formation of acylmethyldinitroquinolone 82 in reaction of trinitroquinolone 67 with enamines $\mathbf{8 1}$ followed by hydrolysis of an intermediate $\mathbf{8 3}$.

2,3-Dichloro-derivatives 84 and 3-chloro-2-morpholino-derivatives 85 of 1-ethyl-pyrazinium tetrafluoroborates reacting with the anions of active carbonyl compounds $\mathbf{8 6}$ produced tele-substituted products 88 via intermediates 87 (Scheme 38). ${ }^{38}$

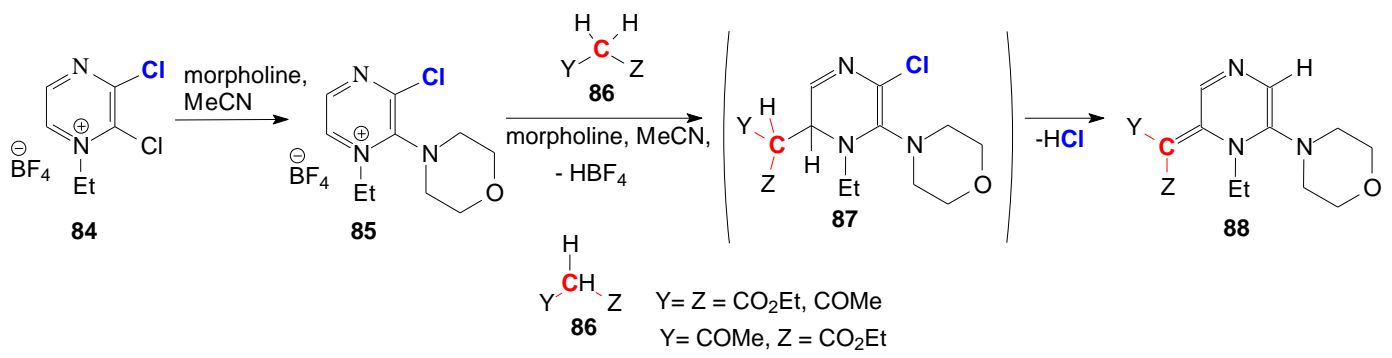

Scheme 38. tele-Nucleophilic substitution of dichloropyrazinium derivatives $\mathbf{8 4}$ and $\mathbf{8 5}$ with active methylene compounds 86 .

Other compounds of type 86 in which $Y=Z=C N$ or $Y=C O O E t, Z=C N$ reacting with pyrazinium compounds $\mathbf{8 4}$ or $\mathbf{8 5}$ afforded predominantly or exclusively products of ipso-substitution of the chlorine atom. 
Copper- and cesium-catalyzed couplings of haloimidazo[1,2-a]pyridines with azoles were studied by Gueiffier et al. ${ }^{39}$ The authors found that the coupling performed in the presence of a copper catalyst led to ipsosubstitution of the halogen while the reaction catalyzed by cesium carbonate afforded the cine-substituted product (Scheme 39).

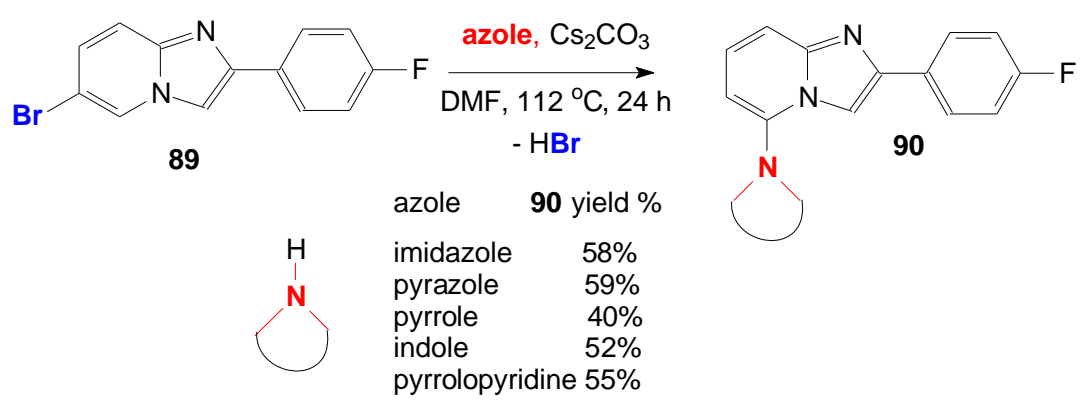

Scheme 39. Cesium catalyzed cine-substitution of heterocycle 89 with $1 H$-azoles.

That was the first example of cine-substitution for imidazo[1,2-a]pyridines. Earlier in this review ${ }^{9,29,33}$ it was described that the 3-nitrogroup in trinitroquinolone $\mathbf{6 7}$ treated with some carbon nucleophiles such as carbanions from 1,3-dicarbonyls, methyl ketones or neutral enamines could undergo cine-substitution with CC-bond formation at the position 4. Later, Nishiwaki et al..$^{40}$ showed that treatment of compound 67 with an excess of aliphatic primary amines could be an effective approach for the formation of the $\mathrm{C}(4)-\mathrm{N}$ bond with the formation of addition products in form of salts $\mathbf{9 2}$ (Scheme 40).

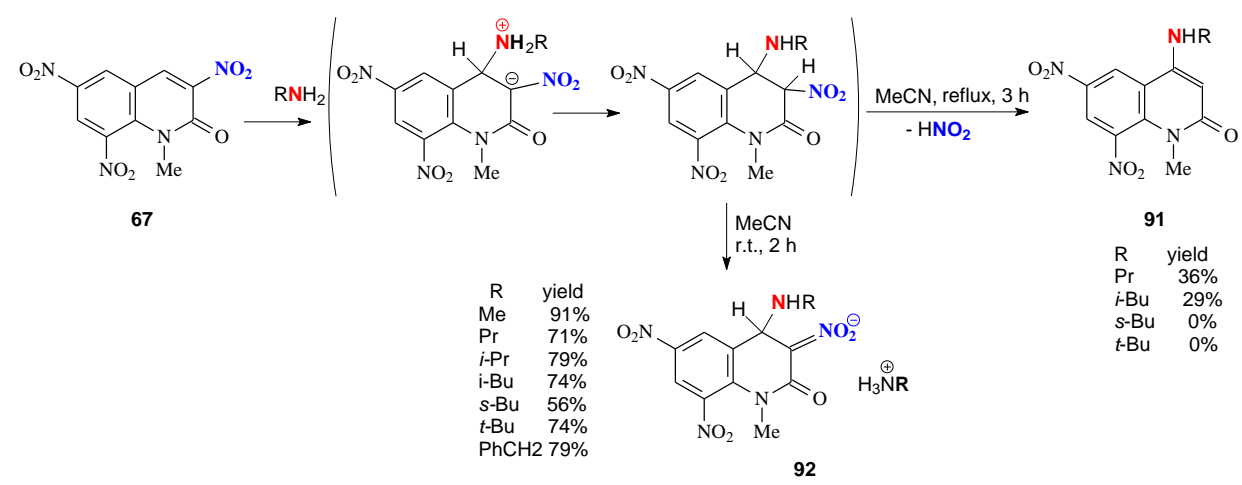

Scheme 40. Reactions of trinitroquinolone 67 with primary amines; the formation of nucleophilic cinesubstitution 91 and addition 92 products.

Unfortunately, cine-substitution of quinolone $\mathbf{6 7}$ to afford products $\mathbf{9 1}$ was possible only for sterically unhindered amines such as propylamine or isobutylamine. Nishiwaki et al. ${ }^{41}$ also described the reaction of trinitroquinolone $\mathbf{6 7}$ with nitroalkanes in the presence of triethylamine, demonstrating another new tool for regioselective $\mathrm{C}(4)-\mathrm{C}$ bond formation on quinolone $\mathbf{6 7}$ skeleton. High yields of cine-substitution products $\mathbf{9 3}$ (Scheme 41) were observed for the reactions performed at $80^{\circ} \mathrm{C}$.

The reactions with nitroalkanes conducted at room temperature afforded cine-products 93 in much lower yields. Here, instead of cine-substitution, only addition-product $\mathbf{9 4}$ was formed in a moderate yield. Continuing their investigations of the reactions of di- and tri-nitro-1-methyl-2-quinolones with nucleophiles, 
Nishiwaki et al. ${ }^{38}$ found for the first time that dinitroquinolone derivative 95 reacted with a carbanion derived from acetylacetone in a similar way as trinitroquinolone 67 to give cine-substitution product 96 (Scheme 42 ).

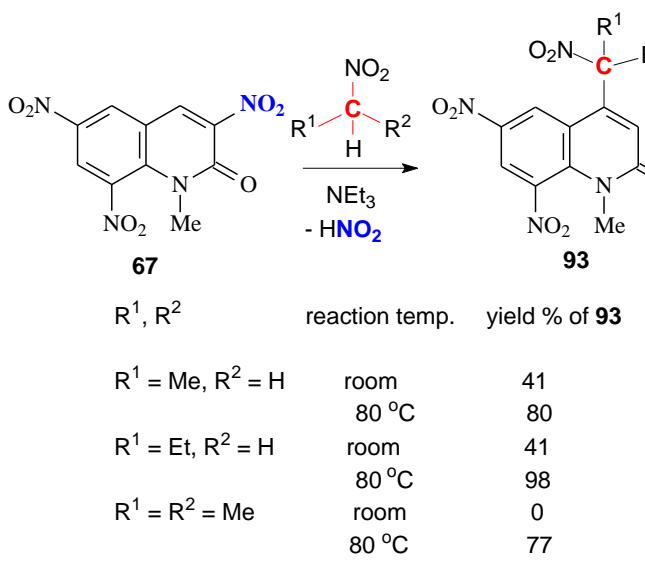

Scheme 41. Effect of the reaction temperature on cine-substitution of trinitroquinolone $\mathbf{6 7}$ with the formation of 1-methyl-4-nitroalkyl-6,8-dinitro-2-quinolones 93.

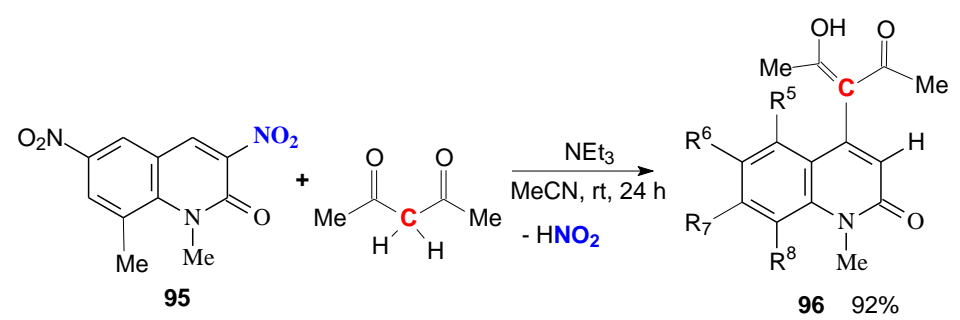

Scheme 42. cine-Substitution of 1,8-dimethyl-3,6-dinitro2-quinolone 95 with carbanion derived from acetylacetone.

Other dinitroquinolones did not follow this reaction pattern. The authors assumed that the high reactivity of compound $\mathbf{9 5}$ was caused by steric interactions between the two methyl groups in positions 1 and 8 in the starting material. To check this assumption, they used a small nucleophile such as cyanide instead of a bulky acetylacetone anion in the reaction with quinolone 95. The reaction with potassium cyanide in acetonitrile at $60{ }^{\circ} \mathrm{C}$ resulted in the formation of a complex mixture of compounds. Among others, the mixture contained cine-substitution product $\mathbf{9 7}$ and dimeric compound $\mathbf{9 8}$. Under optimized reaction conditions, the total yield of compounds $\mathbf{9 7}$ and $\mathbf{9 8}$ was $94 \%$ while the yield of cine-product 97 reached $80 \%$ (Scheme 43).
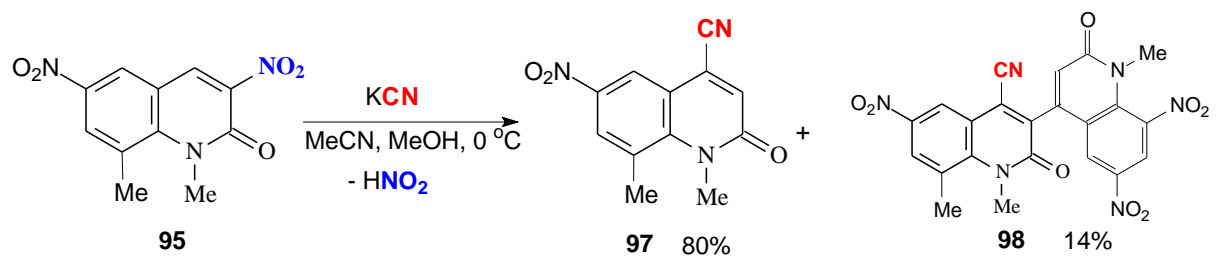

Scheme 43. The highest yields of cine-substituted 97 and dimerized 98 products in the reaction of dinitroquinolone 95 with cyanide. 
When trimethylsilyl cyanide/cesium fluoride was used instead of potassium cyanide, the yield of cineproduct 97 increased up to $90 \%$ while dimerized compound 98 was not present in the post-reaction mixture. Considering the results of the reactions of dinitroquinolone $\mathbf{9 5}$ with acetylacetone anion and with cyanides, it appeared that the presence of a methyl substituent in position 8 was crucial for the occurrence of cinesubstitution. Maes et al. described ${ }^{43}$ a reaction of pyridazinones with Grignard reagents which led to the formation of anionic $\sigma^{H}$ adducts. The adducts were then quickly quenched with electrophiles to avoid elimination and afforded cine-substitution products (Scheme 44).

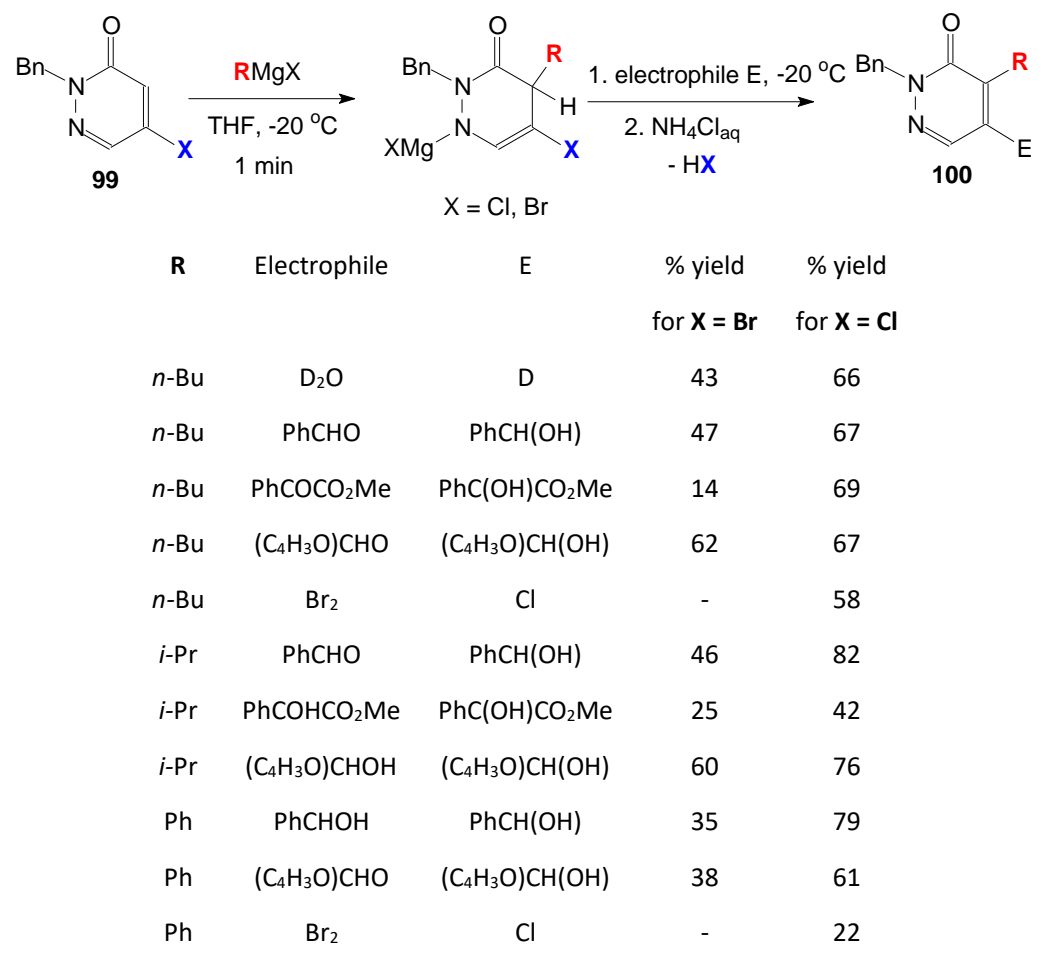

Scheme 44. Examples of cine-substitution of 5-halopyridazin-3-ones 99.

A mechanism of the reaction was proposed (Scheme 45).

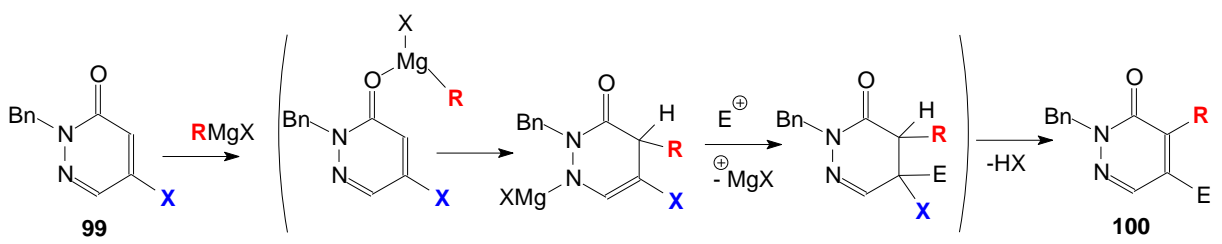

Scheme 45. Plausible mechanism of compound $\mathbf{1 0 0}$ formation as the result of cine-substitution of pyridazine derivative 99.

The authors performed an experiment in which pyridazine derivative 99 was treated with Grignard reagents and then with benzoyl chloride as an electrophile. With this electrophile, an elimination step leading to aromatization did not occur and the reaction stopped at the formation of an addition product. Berezin et al. reported $^{44}$ that a bromo derivative of triazine 101 reacting with thiophenes under acidic conditions and then oxidized with $\mathrm{K}_{3}\left[\mathrm{Fe}(\mathrm{CN})_{6}\right]-\mathrm{KOH}$ resulted in the formation of a mixture of OHVS 102 and cine-substitution 103 
product. The use of morpholine instead of an oxidizing agent led to obtaining compound 103 as the only product (Scheme 46).

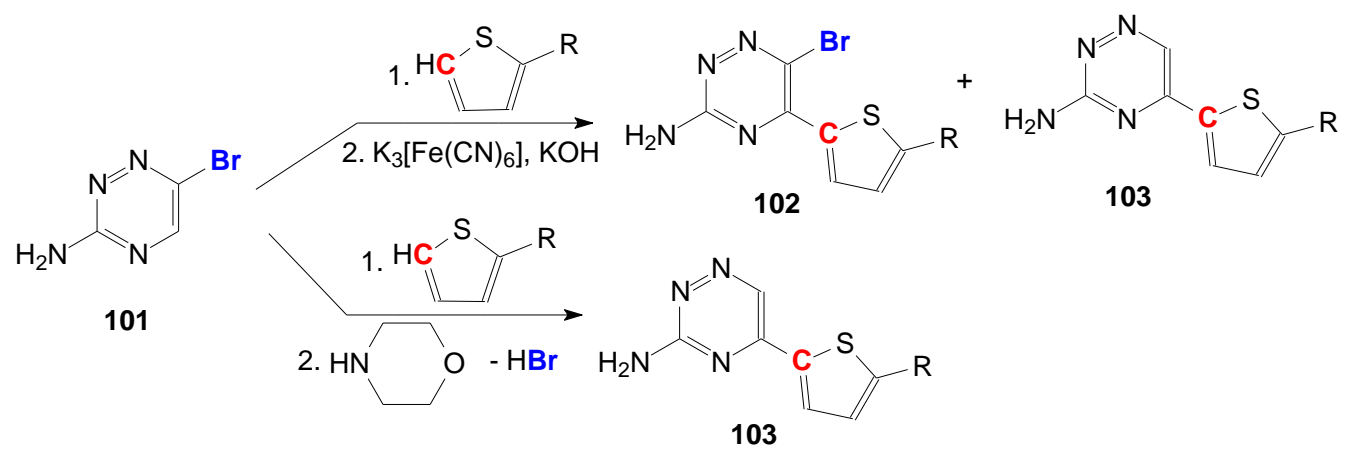

Scheme 46. Formation of cine-substitution products 103 from triazine derivative 101.

\section{2. Five-membered heterocycles with a leaving group at a carbon atom}

Ohta et al. ${ }^{45}$ studied the reaction of chloroalkyl imidazole derivative 104 with diethyl malonate. The reaction conducted in tetrahydrofuran in the presence of sodium hydride produced a mixture of two tele-substituted 105, 106 and one ipso-substituted 107 products. The tele-products predominated in the mixture (Scheme 47).

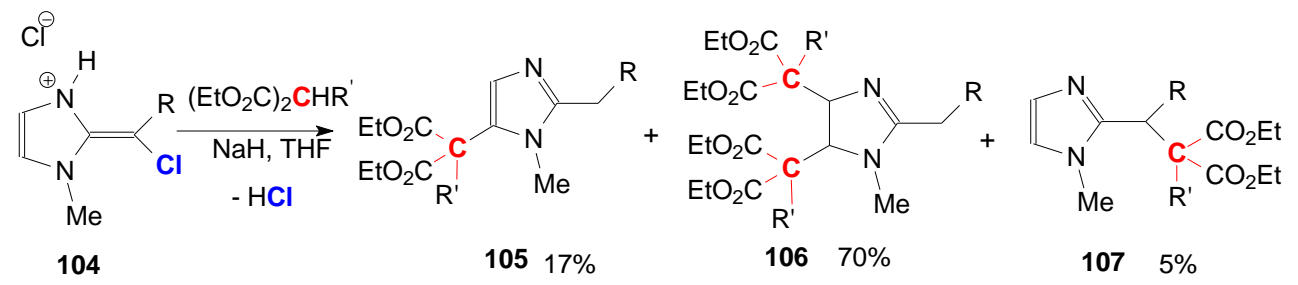

Scheme 47. tele- and ipso-Substitution of chloroalkylimidazole derivative 104.

Several other 2-(1-chloroalkyl)-1-methylimidazoles reacted with malonates in a similar way as compound 104. In all attempts, the yields of both tele-products (like 105 and 106) were high and exceeded the yields of ipso-substituted compounds (like 107). A mechanism of the tele-substitution was discussed and formulated (Scheme 48).

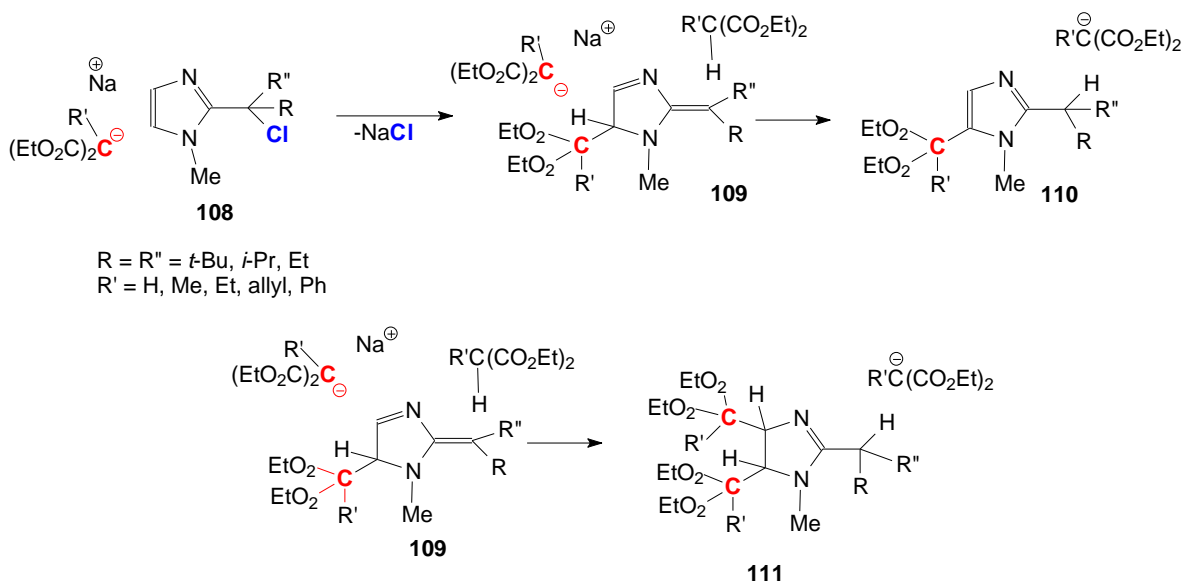

Scheme 48. Postulated mechanism of tele-substitution of chloroalkylimidazole derivatives 108 with sodium malonates. 
Compound 108 also underwent a nucleophilic substitution with Grignard reagents and diethylzinc. With methylmagnesium bromide and with butylmagnesium chloride, the post-reaction mixtures contained tele-112 and ipso-substituted 113 products while the reaction with diethylzinc afforded ipso-product only. Formation of compounds of type 106 (diadducts) was not observed in the reaction probably due to the absence of an acidic proton in the used reagents (Scheme 49).

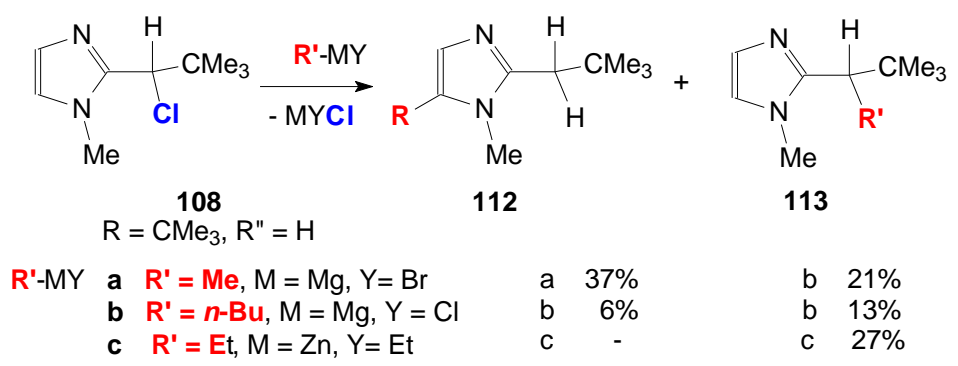

Scheme 49. Reaction of chloroalkylimidazole 108 with Grignard reagents and diethylzinc.

An anomalous course of the reaction similar to cine-substitution was observed by Fasfous et al. ${ }^{46}$ when 3[5-(4-methylpiperazin-1-yl)-3-nitrothien-2-yl]indole (114) was treated with $N$-methylpiperazine to give compound 115 together with the typical ipso-substitution product 116 (Scheme 50).
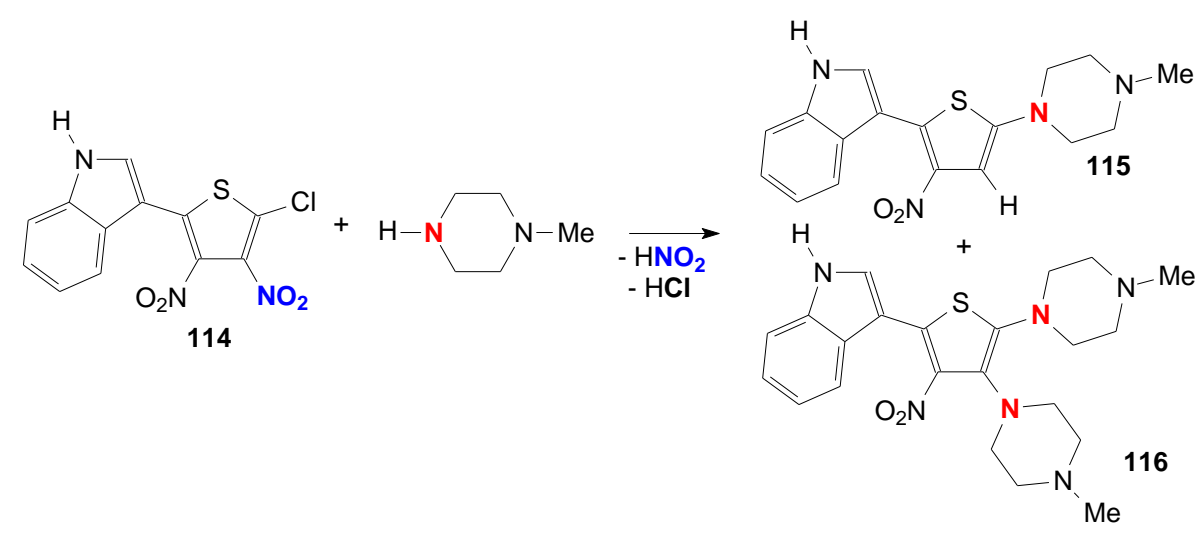

Scheme 50. An anomalous course of reaction of compound 114 with $N$-methylpiperazine.

Reactions of cine- and tele-substitution in thiophene derivatives were briefly discussed in sub-chapter 3.5 (cine- and tele-Substitution Chemistry p.p. 95-96) as a part of the chapter Thiophene Substitution Chemistry written by V. Ryabova and L. Ignatovich ${ }^{47}$ and published on-line in 2014. Examples of cine- and telesubstitutions cited in this sub-chapter are shown in Scheme 51. In compound 117, each of the substituents can play the role of the leaving group in the reaction with arylthiolate. cine-Substitution of compound 120 was not possible and therefore, tele-substitution of an active hydrogen in methyl group occurred. 

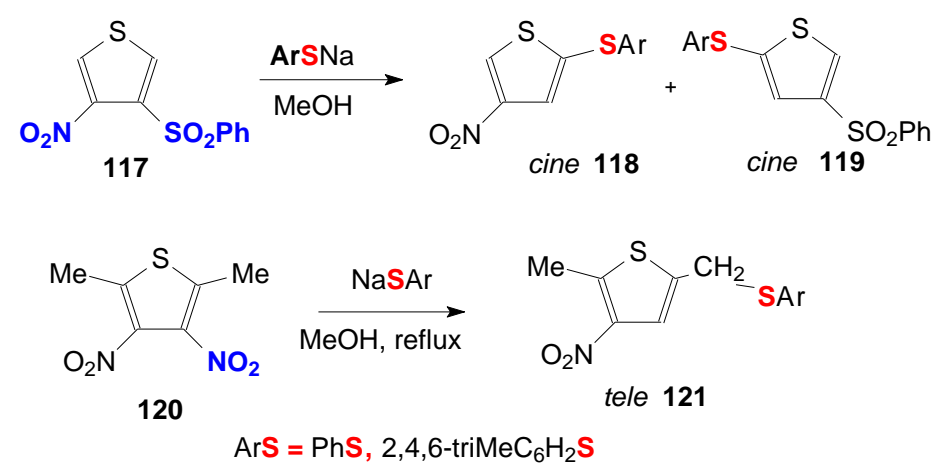

Scheme 51. Nucleophilic cine- and tele-substitution of active thiophene derivatives.

A very interesting first example of nucleophilic cine- and tele-substitutions in the gas-phase has recently been described by Danikiewicz and Zimnicka. ${ }^{48}$ The authors performed reactions of derivatives of nitrothiophene and nitrofuran with some compounds able to form carbanions in an especially adapted mass spectrometer. The formation of $\sigma$-adducts and products of their transformations were observed in reactions characterized by moderate values of proton affinity difference between the $\mathrm{C}-\mathrm{H}$ acid conjugate base and anions from 2nitrothiophene (122) or nitrofuran (123). cine- and tele-substituted compounds were present among the products from nitrothiophene. Reaction pathways were proposed. Further experiments with deuterated acetonitrile (127) revealed that tele-substitution was possible but could not occur via the $1,5-\mathrm{H}$ shift because the intermediate adducts 128 or 130 eliminated only $\mathrm{HNO}_{2}$ as shown in Scheme 52. Elimination of $\mathrm{DNO}_{2} \mathrm{Was}$ excluded in the experiment.

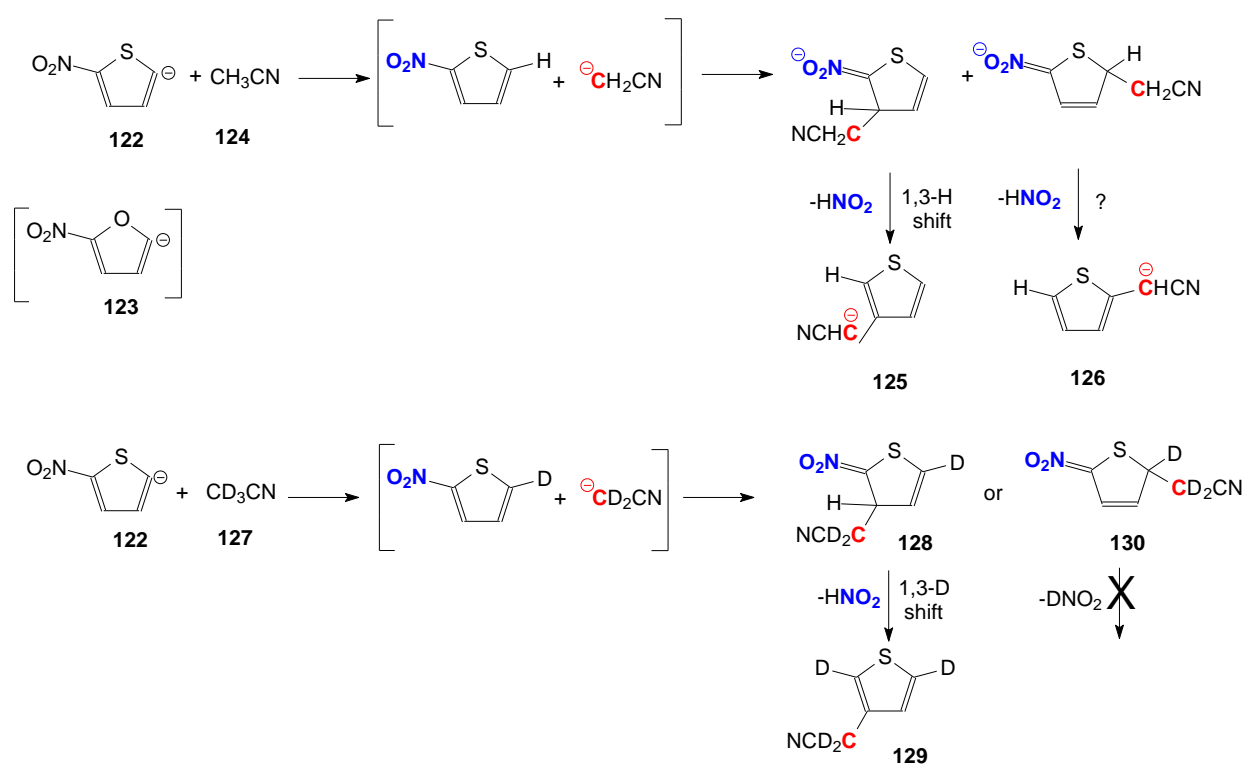

Scheme 52. Proposed pathways of formation of cine-substituted anionic products (125) in gas-phase reaction of 2-nitrophene carbanion 123 with acetonitrile (124).

Several mono- and oligo-bromothiophenes 131 reacted with lithium boranato(tert-butyl) methylphosphide (132) without a catalyst to afford mixtures of two products $\mathbf{1 3 3}$ and $\mathbf{1 3 4}$. The mixtures were formed despite the differences in the number and positions of the bromine atoms in the starting bromothiophene. ${ }^{49}$ It was shown that at least most of the reactions occurred according to cine- and/or tele- 
substitution patterns. The lower yields of products 133 and 134 obtained from 2,5-dibromothiophene (131d) may be interpreted to mean that also in this case, the reaction did not proceed according to the conventional halogen exchange. A reaction of 2,3-dibromothhiophene (131 b) with enantiopure $(S)$-132 performed at $-80{ }^{\circ} \mathrm{C}$ gave completely racemized product 134 while the reaction of 1,3-dibromobenzene with (S)-132 delivered an almost enantiopure product. This finding can be interpreted to indicate that each of the latter reactions followed a different mechanistic pattern. The reaction of 1,3-dibromobenzene involved the formation of an intermediate benzyne derivative. In the case of reaction of 2,3-dibromothiophene, the authors suggested an initial single electron transfer followed by the formation of ions from 2-lithio- or 2,5-dilithiothiophenes and then their combination with a phosphorus reagent (Scheme 53).

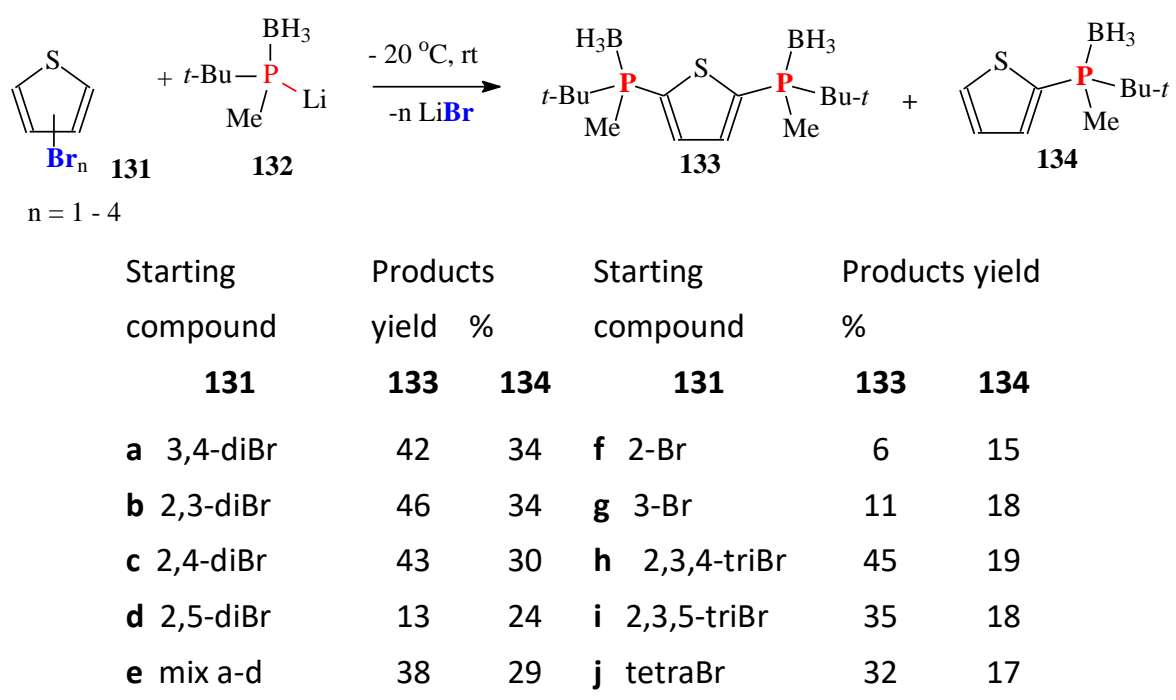

Scheme 53. Reactions of bromothiophenes 131 a-j with lithium ( \pm 1 )-boranato(tert-butyl)methylphosphide (132).

\subsection{Five-membered heterocycles with a leaving group at a nitrogen atom}

A nucleophilic cine-substitution reaction of 1,4-dinitroimidazole and 2-methyl derivative was applied in a synthesis of labelled 4(5)-nitro- $1 H$-imidazole-5(4)-carbonitriles ${ }^{50}$ The starting dinitroimidazoles 136a,b were treated with $\mathrm{KC}^{15} \mathrm{~N}(\mathbf{1 3 6 a})$ or $\mathrm{K}^{13} \mathrm{CN}(\mathbf{1 3 6 b})$ potassium cyanides and gave the respectively labelled carbonitriles 137 (Scheme 54).

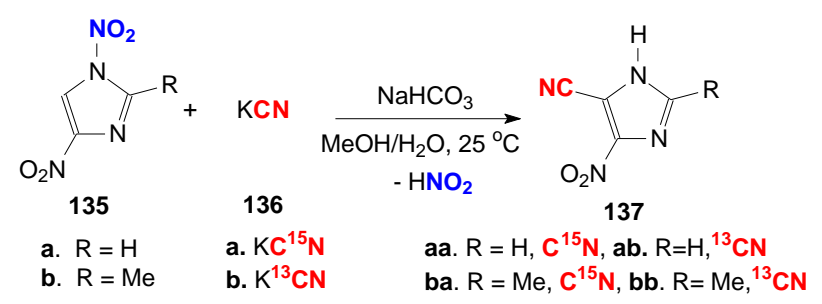

Scheme 54. cine-Substitution of dinitroimidazoles 135a,b with nitrogen-15 (136a) or carbon-13 (136b) KCN.

Detailed analysis of mass spectra confirmed the structures of the products and the labeling. Luo et al. ${ }^{51}$ followed the procedure used earlier by Suwinski and Swierczek ${ }^{50}$ for the preparations of labeled 5-cyano-4nitro- $1 \mathrm{H}$-imidazoles and obtained one new doubly isotopically modified compound: $5(4)-\left({ }^{15} \mathrm{~N},{ }^{13} \mathrm{C}\right) \mathrm{cyano}-4(5)-$ 
nitro- $1 \mathrm{H}$-imidazole, which was then used in the syntheses of the respectively substituted adenine and adenosine derivatives. It is well-known that 1,4-dinitropirazoles react easily with several nucleophiles to form 5-substituted 4-nitropirazoles. Suwinski et al. ${ }^{52}$ showed that the reaction of 1,4-dinitropyrazole (138) with amidines carried out in methanol gave essentially a mixture of oligomethoxynitropyrazoles together with small amounts of the expected respective 5-nitropyrimidines. Under these reaction conditions, methanol was deprotonated by strongly basic amidines and the forming anion then reacted with pyrazole 138 to afford 5methoxy-4-nitropyrazole anion 139. The latter anion ten reacted with compound 138, giving anion 140 . A cascade of similar cine-substitutions resulted in the formation of mixtures of oligomethoxynitropyrazoles (Scheme 55).
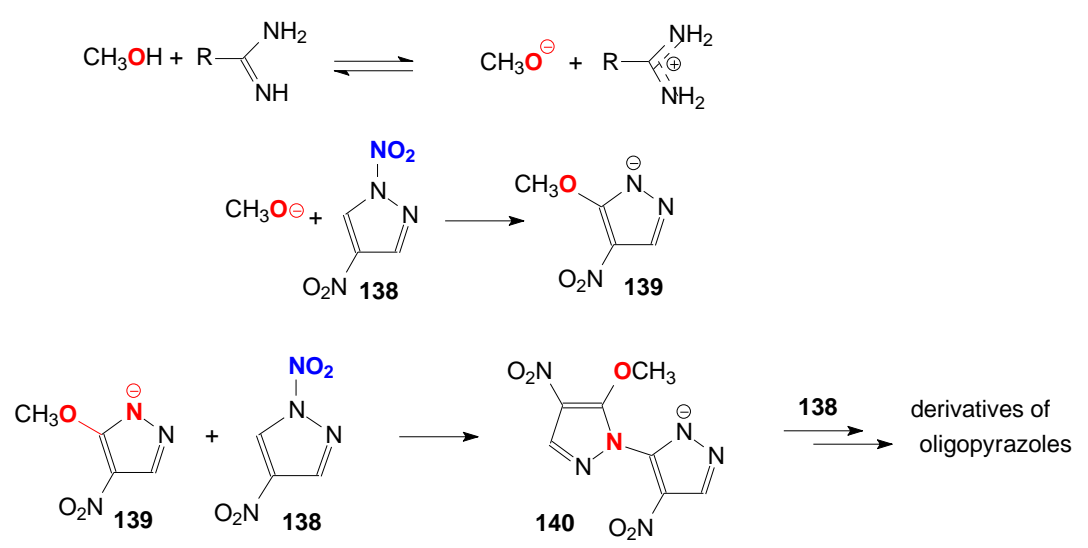

Scheme 55. Formation of oligomethoxynitropyrazoles in reaction of 1,4-dinitropyrazole (138) with methanol in the presence of amidines.

Mityanov et al. ${ }^{53}$ investigated the nucleophilic halogenation of imidazole at carbon atom 2 treated 1-aryl4,5-dimethylimidazole-1H-1-oxide fluoroborat complexes (141) with tosyl halogenides and obtained 1-aryl-2halo-4,5-dimethyl- $1 H$-imidazoles (143). A mechanism involving cine-substitution of intermediate cation 142 with halogen anion was formulated (Scheme 56).

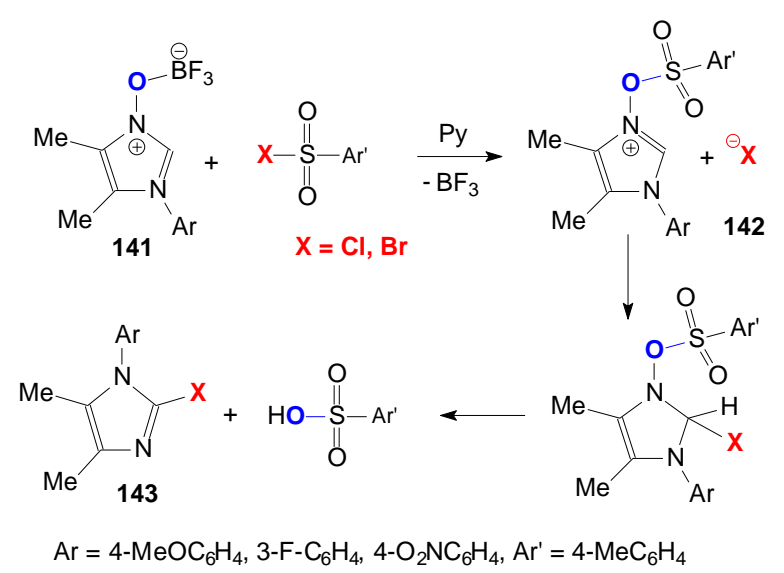

Scheme 56. Nucleophilic halogenation of imidazole $N$-oxides involving cine-substitution.

The authors also proposed that the addition of the halogen anion to the atom C-2 of cation 142 was the rate-limiting step. In 2015, Suwinski ${ }^{54}$ published a review on 1,4-dinitro- $1 H$-imidazoles. A short sub-section 
4.2 of the review was dedicated to nucleophilic cine-substitution of the dinitroimidazoles. Investigations of the reactions of 1,4-dinitropyrazoles with nucleophiles ${ }^{52}$ were continued later by Jędrysiak and Suwiński ${ }^{55}$ who focused on reactions of 3-methyl derivative in this study. It was found that 1,4-dinitro-1H-pyrazole (138) with an excess of anilines afforded ring-opening compounds 144 while 3-methyl derivative 145 gave cinesubstituted compounds 146 in high yield (Scheme 57).

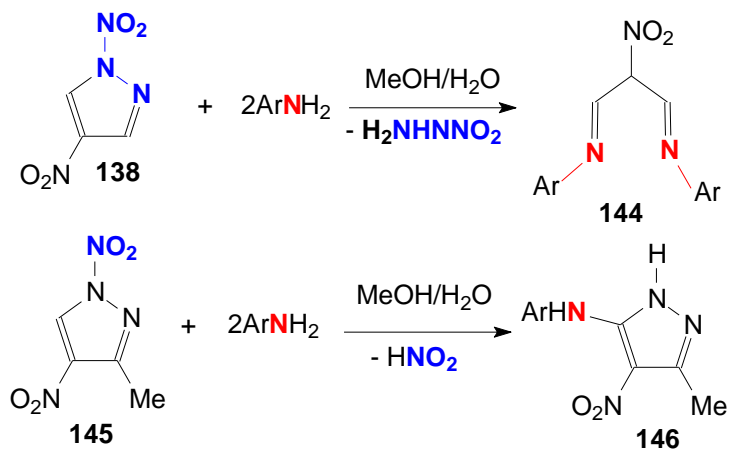

Scheme 57. Different courses of reactions of dinitropyrazoles 138 and 145 with anilines.

Shreeve et al. ${ }^{56}$ looking for energetic compounds with good detonation performance and high thermal stability studied nucleophilic cine-substitution of 1,3 -dinitro- $1 H$-triazole with other $1 H$-azoles in the presence of bases and obtained four derivatives of 5-(azol-1'-yl)-3-nitro-1H-1,2,4-triazoles. The products were fully characterized by NMR and X-ray.

\section{4. cine- and tele-Substitution in Nonaromatic Systems}

As mentioned earlier, cine- and tele-substitution in nonaromatic systems is rare. Furthermore, in certain cases, it is not easy to determine whether a paper addresses reactions occurring in aromatic or nonaromatic system. This characteristic primarily concerns the substitution of complexes of organic compounds with metals. A paper by Rose et al. ${ }^{57}$ providing several references to former articles on cine- and tele-substitutions is a good example of this. Among other references, the paper cited nucleophilic substitutions of $\left[\left(n^{5}\right.\right.$-cyclohexadienyl $\left.) \mathrm{Mn}(\mathrm{CO})_{3}\right]$ complexes 148. It was shown that the mechanisms of the reactions were very similar to those described for $\left[\left(\eta^{6}\right.\right.$-arene $\left.) \mathrm{Cr}(\mathrm{CO})_{3}\right]$ complexes 147 . The similarity can be easily understood by taking into account the reversible addition-elimination of hydride anion between the complexes (Scheme 58).

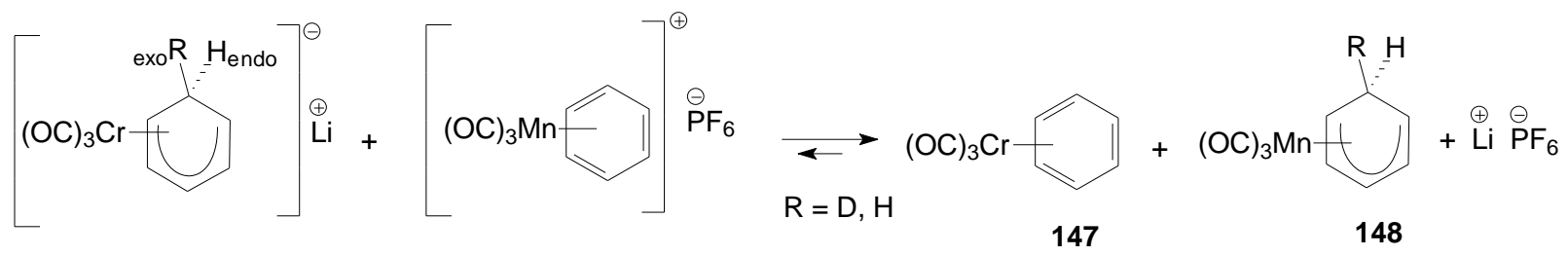

Scheme 58. Reversible addition-elimination of hydride anion between complexes.

Due to the similarity of cine- and tele-substitution mechanisms of cyclohexadienylmanganese and arene chromium complexes, the reactions of cyclohexadienylmanganese complexes are treated here as the reactions of aromatic systems. 
Sanjay et al. ${ }^{58}$ described the synthesis of unsymmetrical allyl chlorides from allyl alcohols or baminoalcohols using cyanuric chloride in dimethylformamide. In some cases, ipso-substitution was accompanied by tele-substitution. Ratios of the ipso- to tele-substitution depended on the positions, and electronic and steric effects of substituents. Only the abstract of this article was available to the author of the present review. The overall picture of the reaction is given in Scheme 59.
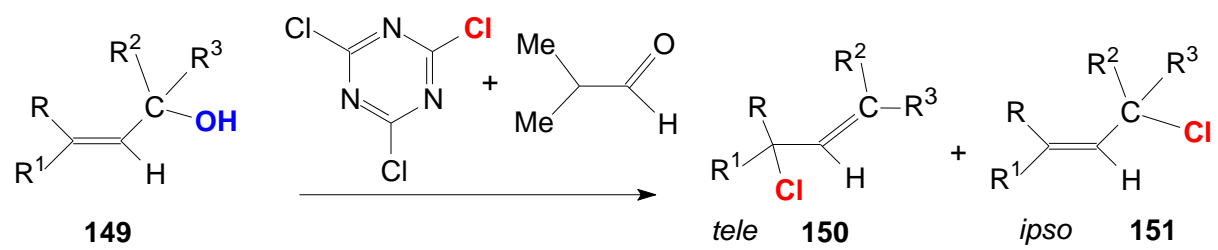

Scheme 59. Formation of allyl chlorides in tele- and ipso-substitution of allyl alcohols.

Matsuda et al. ${ }^{59}$ studied rhodium-catalyzed cross-coupling of alkenyl halides with arylboron compounds. They observed mono- and double-arylation of 1,2-dichloroethene with arylboronic esters in the presence of rhodium(I) catalyst. To gain some more information on the course of the studied reaction, the authors coupled $\alpha$-bromostyrene (152) with phenylboronic acid and obtained a mixture of 1,1-diphenylethene $(153)$ and $(E)$ stilbene (154) in the ratio of 4:1 with combined yield of $46 \%$ (Scheme 60 ).

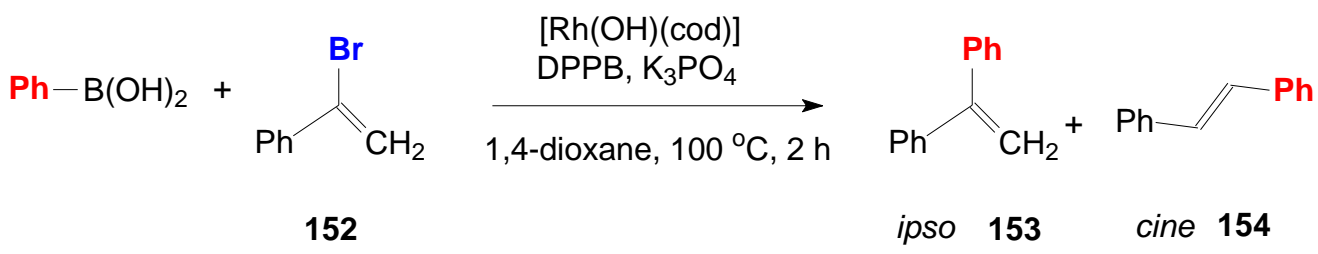

Scheme 60. Arylation of bromostyrene $\mathbf{1 5 2}$ with phenylboronic acid in the presence of rhodium catalyst.

The formation of product 154 could indicate the addition/ $\beta$-elimination mechanism of arylation (Scheme 61).

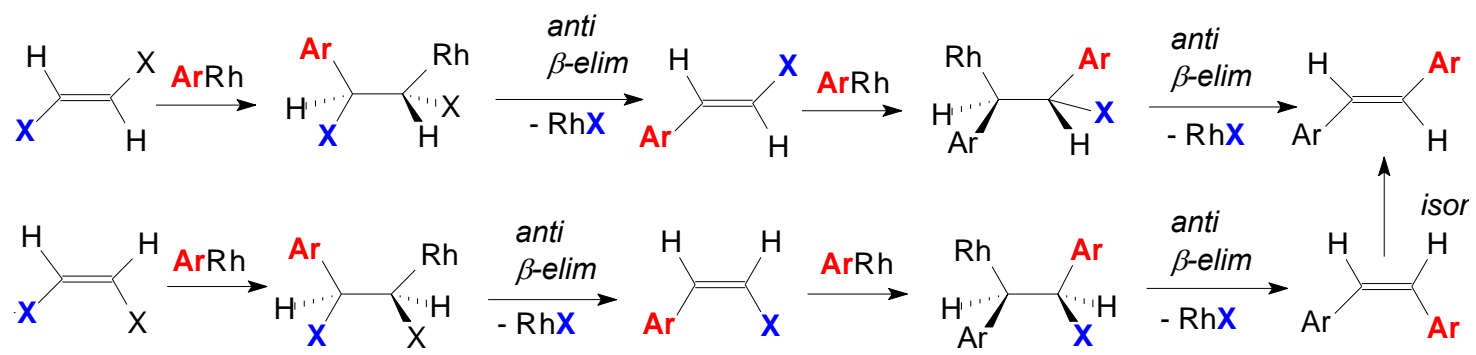

Scheme 61. Proposed mechanism for rhodium(I)-catalyzed arylation of 1,2-dihaloethenes.

Such mechanism was earlier proposed for the known rhodium-catalyzed cine-substitutions of alkenyl sulfones and alkenyl acetates.

Dinesh et al. ${ }^{60}$ described a new original stereo-controlled synthesis of triquinane framework. In the course of the investigations, compound $\mathbf{1 5 5}$ underwent Michael addition with malonic ester to afford Michael adduct 
156 in $60-70 \%$ yield accompanied by compound 157 in $10-15 \%$ yield. According to the authors, product 157 was probably formed by cine-rearrangement of the enolic form of compound $\mathbf{1 5 6 .}$

Formally, the conversion of compound $\mathbf{1 5 6}$ into product $\mathbf{1 5 7}$ may also be called tele-substitution. In principle, the reaction does not seem to differ from highly well-known reactions denoted by $S_{N}{ }^{\prime}$ (Scheme 62).
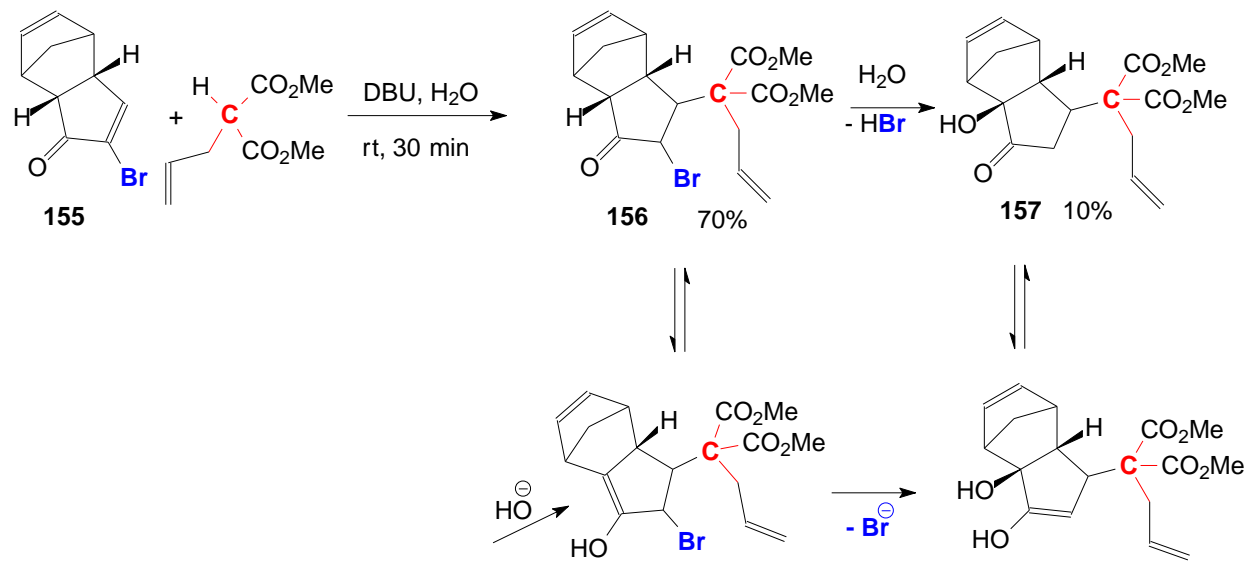

Scheme 62. tele-Substitution of bromine by hydroxyl in a triquinane synthesis.

Rulev et al. ${ }^{61}$ elaborated a new method for the synthesis of push-pull aryloxyenones by nucleophilic cinesubstitution of haloenones with phenolates. Yields of $\alpha$-aryloxyenones depended on nucleophilicity of phenolates and decreased in the following order: 4- $\mathrm{BrC}_{6} \mathrm{H}_{4} \mathrm{O}^{-}, 4-\mathrm{MeO}-\mathrm{C}_{6} \mathrm{H}_{4} \mathrm{O}^{-}, 4-\mathrm{O}_{2} \mathrm{NC}_{6} \mathrm{H}_{4} \mathrm{O}^{-}$. Products were obtained as mixtures of $E$ and $Z$ isomers. Two alternative pathways ( $E-A d$ and $A d-E$ sequences) were considered for the cine-substitution and E-Ad mechanism was found less favorable (Scheme 63).<smiles>[R]C(=O)/C([Y])=C/c1ccccc1</smiles>

158

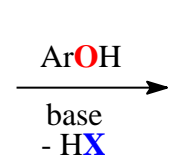<smiles>[R]C(=O)/C=C(/O[AlH2])c1ccccc1</smiles>

$159 \quad 20-64 \%$

158 a: $\mathrm{R}=\mathrm{Me}, \mathrm{Ar}=\mathrm{Ph} ; \quad$ b: $\mathrm{R}=\mathrm{Me}, \mathrm{Ar}=4-\mathrm{BrC}_{6} \mathrm{H}_{4}$,

c: $\mathrm{R}=\mathrm{Me}, \mathrm{Ar}=4-\mathrm{MeOC}_{6} \mathrm{H}_{4}, \mathbf{d}: \mathrm{R}=\mathrm{Me}, \mathrm{Ar}=4-\mathrm{O}_{2} \mathrm{NC}_{6} \mathrm{H}_{4}$,

e: $\mathrm{R}=\mathrm{Ph}, \mathrm{Ar}=4-\mathrm{MeOC}_{6} \mathrm{H}_{4}$

Scheme 63. cine-Substitution of haloenones with phenolates.

A successful experiment with addition of 4-methoxyphenolate $\mathbf{1 6 1}$ to terminal bromoenone $\mathbf{1 6 0}$ led to isolation of 8 -4-methoxyphenoxy- $\alpha$-bromoenone (162) in $50 \%$ yield which under action of a base eliminated hydrogen bromide to afford $\beta$-aryloxyenone 163 . This result pointed out to Ad-E pathway as the most probable mechanism in the studied synthesis of $\alpha$-aryloxyenones (Scheme 64).

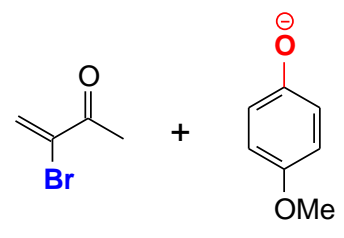

160

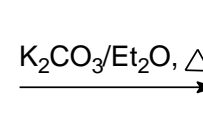
1

Scheme 64. Addition of 4-methoxyphenolate ion (161) to terminal bromoenone (162). 


\section{References}

1. Rose-Munch, F.; Gagliardini, V.; Renard, C.; Rose, E. Coord. Chem. Rev. 1998, 178-180, $249-268$. https://doi.org/10.1016/S0010-8545(98)00078-2

2. Pape, A. R.; Kaliappan, K. P.; Kundig, E. P. Chem. Rev. 2000, 100, 2917-2940. https://doi.org/10.1021/cr9902852

3. Suwiński, J.; Świerczek, K. Tetrahedron 2001, 57, 1639-1662. https://doi.org/10.1016/S0040-4020(00)01067-X

4. Rose-Munch, F. Rose, E. Eur. J. Inorg. Chem. 2002, 1269-1283. https://doi.org/10.1002/1099-0682(200206)2002:6<1269::AID-EJIC1269>3.0.CO;2-9

5. Astru, D.; Rose-Munch, F.; Rose, E. Modern Arene Chemistry, Arenetricarbonylchromium Complexes: Ipso, Cine, Tele Nucleophilic Aromatic Substitutions eds. Wiley-VCH Verlag GmbH \& 2002, Chapter 11.

6. He-Chun, L.; Mei-Hua, S.; Li, Y.; Chao-Zhong, Li. Chin. J. Org. Chem. 2003, 23, 944-949.

7. Mąkosza, M.; Wojciechowski, K. Chem. Rev. 2004, 104, 2631-2666. https://doi.org/10.1021/cr020086+

8. Peng, Y.; Li, W.-D. Z. Eur. J. Org. Chem. 2010, 6703-6718. https://doi.org/10.1002/ejoc.201000557

9. $\quad$ Srivastav, N.; Singh, R.; Kaur V. RSC Adv. 2015, 5, 62202-62213. https://doi.org/10.1039/C5RA09960A

10. Mesganaw, T. T.; Garg N. K. Org. Process Res. Dev. 2013, 17, 29-39. https://doi.org/10.1021/op300236f

11. Nishiwaki, N. Molecules 2010, 15, 5174-5195. https://doi.org/10.3390/molecules15085174

12. Tisler, M. Acta Chim. Slov. 2011, 58, 9-13.

13. IUPAC Compendium of Chemical Terminology Gold Book Version 2.3.3. 2014, 274, 1515.

14. Bradac, J.; Furek, Z.; Janezic, D.; Molan, S.; Smerkolj, I.; Stanovnik, B.; Tisler, M.; Vercek, B. J. Org. Chem. 1977, 42, 4197-4201.

https://doi.org/10.1021/jo00862a005

15. Giannopoulos, T.; Ferguson , J. T.; Wakefield, B. J.; Varvounis, G. Tetrahedron 2000, 56, 447-453. https://doi.org/10.1016/S0040-4020(99)01012-

16. Monk, K. A.; Siles, R.; Pinney, K. G.; Garner, C. M. Tetrahedron Lett. 2003, 44, 3759-3761. https://doi.org/10.1016/S0040-4039(03)00751-2

17. Mąkosza, M.; Varvounis, G.; Surowiec, M.; Giannopoulo, T. Eur. J. Org. Chem. 2003, 3791-3797. https://doi.org/10.1002/ejoc.200300223

18. Błażej, S.; Kwast, A.; Mąkosza, M. Tetrahedron Lett. 2004, 45, 3193-3195. https://doi.org/10.1016/j.tetlet.2004.02.133

19. Mąkosza, M.; Lobanova, O.; Kwast A. Tetrahedron 2004, 60, 2577-2581. https://doi.org/10.1016/i.tet.2004.01.038

20. Surowiec, M.; Belekos, D.; Mąkosza, M.; Varvounis, G. Eur. J. Org. Chem. 2010, 3501-3506. https://doi.org/10.1002/ejoc.200901303

21. Epishina, M. A.; Kulikov, A. S.; Ignatev, N. V.; Schulte M.; Makhova, N. N. Mendeleev Commun. 2015, 25, 41-43. https://doi.org/10.1016/i.mencom.2015.01.015 
22. Tiwari, R.; Miller, P. A.; Chiarelli, L. R.; Mori, G.; Šarkan, M.; Centárová, I.; Cho, S.; Mikušová, K.; Franzblau, S. G.; Oliver, A. G.; Miller, M. J. ACS Med. Chem. Lett. 2016, 7, 266-270.

23. Kim, S. Y.; Lim, J. W.; Nam, K. C.; Kim, J. N. Bull. Korean Chem. Soc. 2016, 37, 1522-1525. https://doi.org/10.1002/bkcs.10880

24. Lin, H.; Yang, L.; Li, C. Organometallics 2002, 21, 3848-3849. https://doi.org/10.1021/om020466l

25. Wang, T.; Zhang, H.; Han, F.; Long, L.; Lin, Z.; Xia, H. Chem. Eur. J. 2013, 19, 10982-10991. https://doi.org/10.1002/chem.201301398

26. Tsukada, N.; Abe, T.; Inoue, Y. Helv. Chim. Acta 2013, 96, 1093-1102. https://doi.org/10.1002/hlca.201200454

27. Ohno, H.; Iwasaki, H.; Eguchi, T.; Tanaka, T. Chem. Commun. 2004, 2228-2229. https://doi.org/10.1039/b410457a

28. Iwasaki, H.; Eguchi, T.; Tsutsui, N.; Ohno, H.; Tanaka, T. J. Org. Chem. 2008, 73, 7145-7152. https://doi.org/10.1021/jo800656a

29. Mesganaw, T.; Nathel, N. F. F.; Garg, N. K. Org. Letters 2012, 14, 2918-2921. https://doi.org/10.1021/ol301275u

30. Tobisu, M.; Chatani, N. Top. Curr. Chem. (Z) 2016, 374:41. https://doi.org/10.1007/s41061-016-0043-1

31. Barroso, S.; Lemaire, S.; Farina, V.; Steib, A. K.; Blanc, R.; Knochel, P. J. Org. Chem. 2016, 81, $2804-2816$. https://doi.org/10.1021/acs.joc.6b00074

32. Nishiwaki, N; Sakashita, M; Azuma, M; Tanaka, C; Tamura, M; Asaka, N; Hori, K; Tohda, Y; Ariga, M. Tetrahedron, 2002, 58, 473-478. https://doi.org/10.1016/S0040-4020(01)01169-3

33. Kozhevnikov, D. N.; Kataeva, N. N.; Rusinov, V. L.; Chupakhin, O. N. Russ. Chem. Bull. (Engl. Transl.) 2004, 53, 1295-1300.

https://doi.org/10.1023/B:RUCB.0000042289.07168.8f

34. Kovalev, I. S.; Kopchuk, D. S.; Zyryanov, G. V.; Rusinov, V. L.; Chupakhin, O. N.; Charushin, V. N. Russ. Chem. Rev. 2015, 84, 1191-1225. https://doi.org/10.1070/RCR4462

35. Torr, J. E.; Large, J.M; Horton, P. N.; Hursthouse, M.B; McDonald, E. Tetrahedron Lett. 2006, 47, 31-34. https://doi.org/10.1016/j.tetlet.2005.10.146

36. Torigoe, K.; Kariya, N.; Soranaka, K.; Pfleiderer, W. Helv. Chim. Acta 2007, 90, 1190-1205. https://doi.org/10.1002/hlca.200790118

37. Asahara, M.; Katayama T.; Tohda, Y.; Nishiwaki, N.; Ariga M. Chem. Pharm. Bull. 2004, 52, 1334-1338. https://doi.org/10.1248/cpb.52.1334

38. Slepukhin, P. A.; Rusinov, G. L.; Charushin, V. N.; Kodess, M. I.; Chupakhin O. N. Russ. Chem. Bull. (Engl. Transl.) 2003, 52, 689-694. https://doi.org/10.1023/A:1023919125480

39. Enguehard, C.; Allouchi, H.; Gueiffier, A.; Buchwald, S. L. J. Org. Chem. 2003, 68, 5614-5617. https://doi.org/10.1021/jo034439w

40. Asahara, M.; Nagamatsu, M.; Tohda, Y.; Nishiwaki, N.; Ariga, M. Arkivoc 2005, (i), 1-6.

41. Asahara, M.; Ohtsutsumi, M.; Ariga, M.; Nishiwaki, N. Heterocycles 2009, 78, 2851-2854. https://doi.org/10.3987/COM-09-11803 
42. Chen, X.; Kobiro, K.; Asahara, H.; Kakiuchi, K.; Sugimoto, R.; Saigo, K.; Nishiwaki, N. Tetrahedron 2013, 69, 4624-4630.

https://doi.org/10.1016/i.tet.2013.04.008

43. Verhelst, T.; Verbeeck, S.; Ryabtsova, O.; Depraetere, S.; Maes, B. U. W. Org Lett 2011, 13, 272-275. https://doi.org/10.1021/ol102703w

44. Berezin, M. V.; Rusinov, G. L.; Charushin, V. N. Russ. Chem. Bull. (Engl. Transl.) 2014, 63, 1359-1363. https://doi.org/10.1007/s11172-014-0603-x

45. Ohta, S.; Sato, K.; Kawasaki, I.; Yamaguchi, Y.; Nishio, S.; Yamashita, M. J. Heterocycl. Chem. 2004, 41, 335-341. https://doi.org/10.1002/ihet.5570410305

46. Fasfous, I. I.; El-Abadelah, M. M.; Sabri, S. S. Lett. Org. Chem. 2011, 8, 712-716. https://doi.org/10.2174/157017811799304124

47. Ryabova, V.; Ignatovich, L. Thiophenes 2014, 39, 43-108.

48 Zimnicka, M.; Danikiewicz W. J. Am. Soc. Mass Spectrom. 2015, 26, 1191-1203. https://doi.org/10.1007/s13361-015-1122-1

49. Imamoto, T.; Horiuchi, Y.; Tamura, K.; Sugiya, M.; Masu H. Org. Lett. 2016, 18, 4132-4135. https://doi.org/10.1021/acs.orglett.6b02094

50. Suwiński, J.; Świerczek, K. J. Label. Compd. Radiopharm. 2002, 45, 795-801. https://doi.org/10.1002/ilcr.607

51. Luo, M.; Schramm, V. L.; Singh, V.; Taylor, E. A. J. Am. Chem. Soc. 2007, 129, 8008-8017. https://doi.org/10.1021/ja072122y

52. Jędrysiak, R.; Sawicki, M.; Wagner, P.; Suwiński, J. Arkivoc 2007, (vi), 103-111.

53. Adiulin, E.I.; Kutasevich, A. V.; Mityanov, V. S.; Tkach, I. I.; Koldaeva, T. Yu. Chem. Heterocycl. Compds. 2015, 51, 500-502.

https://doi.org/10.1007/s10593-015-1727-0

54. Suwiński, J. W. Arkivoc 2015, (i), 97-135.

55. Jędrysiak, R. G.; Suwiński J. W. Arkivoc 2015, (vii), 63-76.

56. Tang, Y.; He, C.; Mitchell, L. A.; Parrish, D. A.; Shreeve, J. M. J. Mater. Chem. A. 2016, 4, 3879-3885. https://doi.org/10.1039/C5TA09803C

57. Jacques, B.; Chavarot, M.; Rose-Munch, F.; Rose, E. Angew. Chem. Int. Ed. 2006, 45, 3481-3484. https://doi.org/10.1002/anie.200600689

58. Sanchita, R.; Tarak, D.; Manabendra, S.; Kumar, C. S.; Sanjay, B. Synth. Commun. 2007, 37, 4367-4370. https://doi.org/10.1080/00397910701578206

59. Matsuda, T.; Suzuki, K.; Miura, N. Adv. Synth. Catal. 2013, 355, 3396-3400. https://doi.org/10.1002/adsc.201300482

60. Dinesh, T. K.; Palani, N.; Balasubramanian, S. Tetrahedron Lett. 2016, 57, 1750-1753. https://doi.org/10.1016/j.tetlet.2016.03.018

61. Mareev, A. V.; Ushakov, I. A.; Rulev, A. Yu. Tetrahedron 2015, 71, 1971-1974. https://doi.org/10.1016/j.tet.2015.02.022 


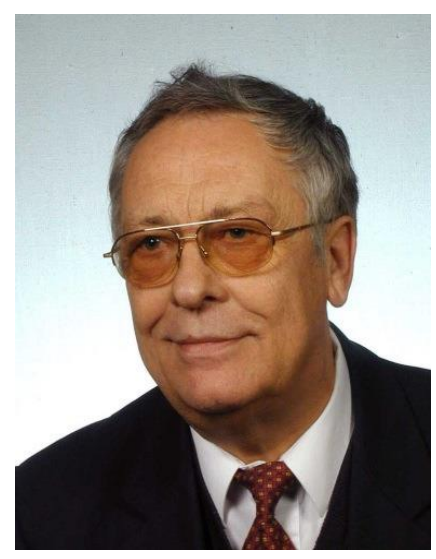

Jerzy Wiktor Suwiński was born in 1939. His M.S. (1961), Ph.D. (1968), D.Sc. (1978) were earned at Silesian University of Technology, Gliwice, Poland where he was employed until 2012. Since 1990, he is a full professor. In 1973-74, he was a post-doc at the University of East Anglia with Prof. A. R. Katritzky. He has lectured several times in Italy, USA and Brazil as a contract or visiting professor. In 2012, he moved to the Centre of Polymer and Carbon Materials of the Polish Academy of Sciences, Zabrze, Poland. His scientific interests lie in the fields of organic synthesis, reaction mechanisms and nitrogen-containing heterocycles. 